\title{
A novel JavaScript MEAN stack approach for real- time nonconformity management in SMEs within Industry 4.0 and Quality 4.0 concepts
}

\section{Aleksandar Đorđević ( $\square$ adjordjevic@kg.ac.rs )}

University of Kragujevac Faculty of Engineering: Univerzitet u Kragujevcu Fakultet inzenjerskih nauka https://orcid.org/0000-0003-2856-6578

Miladin Stefanović

University of Kragujevac Faculty of Engineering: Univerzitet u Kragujevcu Fakultet inzenjerskih nauka Tijana Cvetić

University of Kragujevac Faculty of Engineering: Univerzitet u Kragujevcu Fakultet inzenjerskih nauka Milan Erić

University of Kragujevac Faculty of Engineering: Univerzitet u Kragujevcu Fakultet inzenjerskih nauka Milan Mišić

Higher Technical School of Professional Studies Zvečan

\section{Research Article}

Keywords: Quality 4.0, nonconformity report, nonconformity management, open-source solution

Posted Date: June 21st, 2021

DOl: https://doi.org/10.21203/rs.3.rs-256493/v1

License: (c) (i) This work is licensed under a Creative Commons Attribution 4.0 International License. Read Full License 


\section{Abstract}

Nonconformity detection and reporting are evergoing issues in quality management practice and theory. Throughout industrialisation, and consequently, in the Industry 4.0 period, manufacturing organisations strived to reduce nonconformities to the minimum level and reach zero-defect production. Nowadays, it is feasible to overcome this issue by incorporating adequate information and communication technologies and edge devices for nonconformity reporting, and nonconformity classification. These assumptions were a starting point for the research presented in this paper. This paper aims to propose a software solution for nonconformity detection and preventive and corrective actions definition that expands the utilisation of edge devices in compliance with Industry 4.0 and Quality 4.0 paradigms. Presented software solution design is based on JavaScript programming language and its ability to be implemented in all software solution's tiers through interconnected frameworks (MongoDB, Express.js, Angular, and Node.js) in a MEAN stack. The developed solution is implemented in three small and medium enterprises. Initial results show several benefits in increasing nonconformity detection and reporting, increasing employee participation in preventive and corrective actions definition and improved quality management system. The number of corrective and preventive actions was taken based on the assistance of the prediction module. All nonconformities were related to specific sections of ISO 9001:2015 standards so that quality managers and managers in the companies could have insight into the sources of the issues and the foundation for defining different managerial actions. When comparing the newly developed solution and other affordable solutions, it was determined that the new solution has a higher operating velocity if there is a significant increase in the volume of queries towards databases. The paper's main contribution reflects in the software solution's presentation intended for affordable identification and nonconformities massive workload reporting, integrated with other software modules for quality analysis, prediction, and problem-solving. In this way, it is possible to obtain horizontal scalability and richness of the proposed software solution for smart enterprises focused on World Class manufacturing and lean manufacturing. Furthermore, it is possible to achieve a shorter time for nonconformities elimination and better compliance with the ISO 9001: 2015 standard. The presented solution usage contributes to all participants involved in the organisation of production and production itself, through (1) involvement of all employees; (2) digitalisation and improvement of existing nonconformity reporting systems; (3) improvement of nonconformity perception; (4) improved awareness and evaluation of employer's contribution to nonconformity management; (5) making the technology easy to use. The research's novelty lies in presented solution utilisation for real-time nonconformity classification and proactive and corrective measures proposals through communication and prediction modules.

\section{Introduction}

The primary objectives of all modern manufacturing organisations (MOs), that want to remain competitive, are achieving higher quality and meeting customer demands. These objectives may be achieved if the organisational processes and products are continually monitored, controlled and improved, i.e. if the quality management (QM) is performed. However, although QM initiatives are implemented and applied, quality requirements derived from the turbulent market have created 
challenging issues to be overcome by applying only classical QM measures (Jia et al. 2017). These challenging issues include, above all, a lack of production time, raw materials, and adequate human resources (Ivanov 2016), and, as such, may produce nonconformity (NC) events. In such circumstances, nonconformity management (NCM) and continuous improvements represent important tasks of QM in MOs. While facing possible NCs, MOs can acquire and use information and communication technologies (ICT) that facilitate information and enable the implementation of effective proactive and corrective QM measures. Therefore, the minimised overall costs and increased information flow within manufacturing processes form the smart manufacturing concept foundations. Accordingly, smart MO concept and smart QM could be achieved with the ubiquitous Industry 4.0 (14.0) and Quality 4.0 (Q4.0) era that involve the use of technologies such as: intelligent detection, Internet of Things (IoT), cloud computing environment, and a large amount of data analytics providing an insight into the data related organisation processes and products (Müller et al. 2018; Moeuf et al. 2018).

This paper primarily intends to demonstrate that technologies from the 14.0 toolset could be implemented in QM and NCM, making an essential step towards 14.0 and Q4.0 convergence. To achieve this goal, authors have developed and implemented an innovative software solution with several advantages affordable for Small and Medium Enterprises (SMEs). Developed software application for mobile devices will enable all employees to report and manage NCs using the steps and directives from ISO 9001:2015 (detection, reporting, decision-making, and the definition of preventive and corrective actions). The main contribution reflects in the presented software solution for affordable identification and massive workload NC reporting, integrated with other software modules for quality analysis and prediction. In this way, it is possible to obtain horizontal scalability and richness of the proposed software solution for smart enterprises focused on World Class Manufacturing and Lean manufacturing. The presented solution usage contributes to all participants involved in the organisation of production and production itself, through (1) involvement of all employees; (2) digitalisation and improvement of existing nonconformity reporting systems; (3) improvement of nonconformity perception; (4) improved awareness and evaluation of employer's contribution to nonconformity management; (5) making the technology easy to use inexpensive and suitable for a wider industry audience.

In the following sections of the paper, the authors have presented the concept of NCM in 14.0 (Sect. 2), with the proposed general software components and their relationships design (Sect. 2.1) and process flowchart (Sect. 2.2) included. Additionally, mobile devices, cloud and Q4.0 application trends, JavaScript, and Typescript based solution have been considered. Lastly, the authors have presented a specific case of developed software infrastructure, with the possible benefits that the solution has provided (Sect. 3 and 4).

\section{Theoretical Background And Literature Review 2.1 Industry 4.0 and Quality 4.0 concepts}


14.0 concept is based on developing new technologies such as networking, smart assets, and monitoring (Trappey et al. 2017; Schumacher et al. 2019). 14.0 ought to improve the information flow throughout the entire organisation, enabling better control and operations to be adapted in real-time (Moeuf et al. 2018) to respond to stakeholders' expectations while maintaining a competitive advantage. According to Trappey et al. (2017), 14.0 represents a necessity to maintain competitiveness in an uncertain industrial environment. The 14.0 industry's goal is to promote the production model that is flexible in terms of products and services that are produced and integrated with real-time communications between all the related parties and facilities when performing manufacturing processes (Zhou et al. 2015).

To become agile, manufacturers have to distribute intelligence and decision-making processes. For this purpose, it is necessary to incorporate Internet-based e-manufacturing for information/data management and quality assurance since e-Manufacturing enables a higher level of automation of manufacturing business information than a physical one used before (Cheng et al. 2008). In this way, work in MO is being changed since it can be characterised as: digitalised - all manufacturing information are digitalised, stored and accessed through Internet; globalised - global access to information, regardless of the physical location; mobile - enables accessing information from any place; collaborative - through data sharing companies may crate joint development teams with members from different geographic areas; and immediate-by approaching internet-based platforms it is possible to have latest manufacturing-related information. Based on these assumptions, the system "e-ProMan" has been presented (Katchasuwanmanee et al. 2016). This predictive system acquires real-time data from both inside and outside the SME environment to analyse the correlation between workflow (obtained through process and production schedule data), data flow (acquired using temperature sensors), and energy flow (gettered with power logger equipment) to provide accurate real-time decision-making.

Tuertmann (2016) stated that the increasing volume of the information being collected and the datadriven design led to the inevitable situation of intelligent organisation management. Furthermore, Wee et al. (2015) claimed that the following four technological clusters drove 14.0: 1) connectivity and computational power, 2) artificial intelligence and analytics, 3) interaction between human and machines, and 4) conversion from digital to a physical world. As observed by Geissbauer et al. (2016), 14.0 focused on the comprehensive digitalisation of all assets and resources and their integration within the digital ecosystems while providing them with the possibility to produce, analyse and communicate data. Consequently, 14.0 established a new control and management level through the entire value chain in the product life cycle (Koh et al. 2019). In addition, 14.0 improved the opportunity for the realisation of the dynamic self-coordinated value chain in real-time through the coherence of all involved assets, systems, and people. Based on claims of Hermann et al. (2016), the main 14.0 assumptions are: 1) real-time capability, 2) service orientation, 3) modularity, 4) interoperability, 5) virtualisation, and 6) decentralisation. While achieving 14.0, it is necessary to encompass eight precondition objectives which are: 1) system standardisation, 2) establishment of reliable and extensive broadband infrastructure within industrial organisation, 3) efficient management initiatives, 4) security and safety measures, 5) work design and organisation, 6) improved efficiency of resource use, 7) continuing professional development, and 8) regulatory framework establishment (Liao et al. 2017). 
According to Kim et al. (2016), modern industries and intelligent manufacturing require specific 14.0 technologies in the context of loT to maximise the quality of information collected and to meet the needments for decision formulation in real-time. Industrial loT (IloT) features, including sensors, middleware and storage systems, networking technologies, software, and applications, are combined to develop solutions that can gain insight and improve the capability to monitor and control enterprise processes and assets (Lampropoulos et al. 2018). Observing IloT services and applications, it can be stated that they represent vital applications for more effective planning and controlling of manufacturing processes and systems (Gilchrist 2016). Furthermore, through the various interconnected edge devices that can interact and communicate, IloT may decentralise decision-making and analytics, leading to realtime responses and reactions (Mocnej et al. 2021). Thus, the general availability, monitoring and control of MO processes are enhanced (Gilchrist 2016).

In the 14.0, the quality concept has been broadened, and it includes personalised service quality and personalised production. In 14.0, product quality goes along with design, safety, and service quality (Bonilla et al. 2018). Quality goals have evolved along with the phases of revolution in industrialisation. According to the literature sources (Foidl and Felderer 2015), crucial preconditions for sustainable economic success in modern manufacturing companies are focus on a QC, QM and QMS, respectively. Through smart factory, cyber-physical system (CPS), loT and cloud computing, there is a promising opportunity for any company to adopt Q4.0 and to produce high-quality products and to remain competitive and satisfy the permanently growing customer demands. Therefore, quality goals may progress in line with QC, quality assurance and QM. For instance, IloT technologies can provide improved connectivity systems, services, and physical objects, allowing object-to-object communication and data distribution. Thus, in various industrial applications, QC, quality assurance and QM can be achieved by IloT, since one of the cornerstones of the lloT is the automatic identification technology (Farooq et al. 2015). With an intensive advance toward IloT technologies, data is becoming more available and ubiquitous, culminating with the big data analytics environment (Manyika et al. 2011). In such an environment, datasets are of a much larger volume and maybe too convoluting for standard statistical tools and methods (Ramasamy and Chowdhury 2020). Having this in mind, for MOs with a myriad of operational and shop-floor data application of modern analytics techniques are crucial for uncovering hidden patterns, undiscovered data interrelationships, market trends, customer requirements, and other favourable business information (Barton and Court, 2012).

Additionally, a CPS, as a mechanism by which physical assets and applications are closely related, enables different segments to collaborate in countless courses to transfer information, apply services such as computational capacities and control algorithms (Gunes et al. 2014). An appropriate solution for storing big data may be found in cloud computing, since the horizontal scalability of cloud computing makes it convenient for MOs, as it grants organisations to commence with minor memory volumes and purchase more resources, merely if there are further higher memory demands (Zhang et al. 2010). Finally, through ICT, consolidated and integrated telecommunications are highlighted, with the profound ability to transmit, store, and manipulate data or information (Hashim 2015). ICT focuses on the information transferring through electronic media like wireless or wired communication standards, representing a 
crucial component in intelligent manufacturing, in production and decision-making processes that heavily rely on the data (Bloom et al. 2014). With innovative connectivity technology, smart computing, and Al, QC and assurance are maximised, while standards and stakeholder requirements are easily met (Schmitt et al. 2020).

Alignment of the QC and QM with the concept of Q4.0 and I4.0 enables enterprise efficiency, performance, innovation and business model enhancement. The QC and QM digitalisation and the influence of that digitalisation on processes, technology, and people represent the main direction towards harmonisation with the 14.0 concept. By gathering and processing quality information, innovative production facilitates computerised audit and context perception with a consequent performance increase. Production and QM may use this novel accumulated data and subsequent information for advanced decision-making and process assessment (Putnik et al. 2015), especially since modern QM attempts to establish high performance with maximum efficiency of all organisation processes (Singh et al. 2012). Therefore, 14.0 provides favourable circumstance for the QM field.

With the expansion of current information technology (IT), organisations may gain straightforward data access and improve the potential for knowledge extraction, increasing the influence of 14.0 (Trentesaux et al. 2016). Some examples of applications include wireless sensors networks (Wang et al. 2016; Li et al. 2017), camera monitoring (Kim et al. 2019), smartwatches (Kolberg and Zühlke 2015), smart glasses (Hao and Helo 2017) and smartphones (Vernim and Reinhart 2016) constitute evidence of successful 14.0 implementation ensuring Q4.0 in production processes. Current technologies introduce several software tools available for QM, specifically for quality inspection and reporting. However, according to the literature, authors argue that these solutions may not be adequate, since they use various devices that may decrease compatibility and performance, and increases system complexity, while raising the question of organisation sensitive information privacy and security (Stergiou et al. 2018). Looking at these solutions from SMEs' angle, they encounter additional implementation costs for the features exceeding their capabilities and requirements (Huang et al. 2013). Since SMEs are mostly limited with defined requirements and smaller budgets, compared to the larger organisations that incorporated complex software systems and solutions, the authors of this paper aimed to explore the feasibility of solution that used smartphones and cloud technology to extend reporting of potential NCs within QM. A further assumption is that SME requires simple, prompt and an inexpensive solution (Ren et al. 2015). These solutions should provide data collection and storage with the use of appropriate infrastructure. Some authors proposed mobile data-collection systems suitable for processing within android and IOS environments (Park 2015) since mobile devices have built-in sensors or the ability to communicate with remote sensors. Mobile devices, with optimal hardware, software, and Application Programming Interfaces (API) (Alcácer and Cruz-Machado, 2019), are being imposed as adequate infrastructure solutions. Since mobile devices can present augmented reality, they have been used as a quality tool for key performance indicators presentation, at each work station in a production organisation (Segovia et al. 2015). 
The graphical illustration of the proposed Q4.0 approach is specified in Fig. 1. The overall objective is to support company efforts to enhance its business processes, technology utilisation, and employee operability through the NCs and overall management process's digitalisation.

The core concept of Q4.0 encompasses eleven axis (components) according to Jacob (2017), including: (1) big data with characteristic of volume, variety, velocity, veracity and transparency; (2) analytics framework that provides descriptive, diagnostic, predictive and prescriptive analysis; (3) connectivity that may enable near real-time feedback from linked edge-devices, people and processes; (4) collaboration through digital messages, description of the activity flows (visual), and social (social) media; (5) app development including mobile applications, platforms, virtual reality, augmented reality, web-client, browser and applications for robot (measurement and manipulation) and machines (CNC, DNC, sensors, etc.); (6) horizontal scalability represents the ability to support a growing and large volume of data, users, devices and analytics globally; (7) management systems that monitor autonomous and connected processes; (8) compliance that among other things includes electronic submission of the compliance/NC reports and automation of flows in the compliance area; (9) quality culture representing among functionally strong cooperation, credibility, and shared responsibility; (10) leadership expressed through quality performances, process ownership, and goal scheduling; and (11) competency considering employees training as one of the most crucial fields for business progress. Therefore, through Q4.0, critical new technologies are affordable and accessible to the broader circle of business organisations, with the provided opportunity to solve long-standing quality challenges and adopt new solutions. Following these suggested axes, it is clear that the primary objective of this paper is a representation of the mobile solution for NCM that is based on a collection of big data, management support, improved communication between employees, and improvement of quality culture as essential toolset elements in the Q4.0 concept.

\subsection{Nonconformity management}

Due to increasing market competition, organisations have adopted QMS like the ISO 9000: International Standard Series. According to the ISO survey, 878664 organisations worldwide have certified ISO 9001:2015 (ISO, 2018). The establishment of tools for prevention of NCs and elimination of their causes represents a mandatory requirement of QMS, and as such, NCM receives sub-process function in the general production process (Závadská and Závadský 2018). NCM should (according to ISO 9001:2015) be implemented during the realisation of all organisational processes, and the organisation should retain documented information about NCs. As specified by standard requirements, documented information can be formulated as a report with a description of NC, undertaken actions, obtained concessions and identified authority. Additionally, the standard requires documented information as evidence of any corrective action results, supplementing the report, as mentioned earlier. By utilising the report, corrective and preventive actions are taken to eliminate identified NCs.

NCs in production may be observed from the viewpoints of many different disciplines, including management, economics, environment, technics or technology (Závadská and Závadský 2018). These facts are derived from a modern industrial organisation's goals, reduce production costs and amount of 
misused raw materials, and increase public awareness of environmental sustainability (Marques et al. 2019, Teixeira et al .2020). Giorgetti et al. (2017) introduced NCs reporting through the holistic model based on Root Cause Analysis and TRIZ method. This model uses Poka-Yoke data collection to create an intelligent database that can track the NCs over time and cluster them by Pareto analysis to manage all business processes NCs. Nikolay (2016) presented reports dealing with NCs management's cost in the QMS by examining the costs for causes elimination. Donauer et al. (2015) presented a model for identifying root causes of NCs, by a Herfindahl Index used as the concentration measurement method.

This paper focuses on NCM, intending to decrease the number of products and processes of NCs and effectively implement proactive and corrective measures adequate for product and process quality improvement. Ideally, NC and waste should be eliminated, whereby Zero-defect production should be achieved. Meaning, it is necessary to implement QC in real-time, enabling NC detection in an early process stage and reducing production waste (Djekic et al. 2011). In general, these requirements could be derived from a standard, a specification, a customer or a stakeholder.

Therefore, the detection and reporting of NC could happen during the audit, inspection, documentation review, product testing, customer complaints, stakeholders feedback, and general observation based on experience (Luca 2015). In addition to the above, NCs can take many forms and types, which may vary depending on the industry type, so that common NCs include: failure to identify issues, failure to define processes, plans, and schedules adequately, process deviations and product defects, deviations from a specification of product characteristic, missed plan, customer and supplier return. Taking into consideration the facts stated, NC data are scattered throughout different organisation systems in heterogeneous formats. Specific ICT solutions with scalable capabilities could be applied to overcome data formating diversity and heterogeneity, creating a real-time informed decision-making environment for NCM through the creation of homogeneous digital NC reports.

NC reporting may present a challenge, including specific details and requirements against the NC, NC description, and it may identify production process weak points that are presumed to have led to the NC (Donauer et al. 2015), enabling process owner to confirm the NC. It may present an initial point for the implementation and step towards 14.0 and Q4.0 concept convergence.

\subsection{Initial presumptions and goals for the current research}

The initial presumptions, based on which the research was conducted, are that a sufficient information flow is highly emphasised for the continuous operating of the advanced manufacturing processes and that, however, unpredicted situations and quality issues have to be often resolved by utilising imprecise and incomplete information. Therefore, it is crucial to perform frequent comprehensive and reliable quality inspections to conduct and deliver defect-free and high-quality processes and products and be competitive. Information from organisation processes is becoming transparent, precise, and complete with quality improvement programs like Six Sigma and Kaizen, encouraging data collection to address quality issues and NCs, and advanced 14.0 automation and computing systems. Although traditional data analytic techniques and tools have been widely and successfully used for quality inspection, new 14.0 
techniques may be applied to mine massive data sets gathered through automated industry architecture. Furthermore, since NCs issues may involve multiple inputs and outputs that are difficult to detect, knowledge discovery in databases (KDD) is needed to perform pattern recognition and inform quality practitioners.

Following the presumptions mentioned above and demands of Q4.0, including NCs reporting, this research's primary intention is to adopt technologies from the 14.0 toolset (such as cloud computing, artificial intelligence, mobile platforms, advanced information technologies) to demonstrate road to possible transition of quality to Q4.0 model. In this research, the authors will be focused on QM and NCM as an essential element of the quality approach. This paper's authors demonstrated that affordable openbased solutions developed on open source technologies could improve the NCM and basic principles of QM. The authors presume that the development of affordable cloud solutions based on MEAN stack may be used for real-time NCM to improve basic principles of QMS such as engagement of people, improvement, and evidence-based decision-making.

NCM comprehends different tools for quality assurance and inspection, which can be enforced in any organisation. There are very few literature sources that are focused on methods for the identification and reporting of NCs. Nevertheless, within the organisations, a significant amount of different problems may occur. One of the possible problems arising is how to identify and report the NCs within the organisation. Even though organisations are faced with different NCs, identification and reporting methods are still not perfect. According to the literature, there are two possible approaches to identify NCs. In the first one, NCs detection is performed by the organisation employees, while in the second one, detection is performed by an external expert (Oakland 2005). According to contemporary literature, all other proposed methods are based on these two approaches. Both of these approaches aimed to reduce the number of NCs and prevent NCs occurrence. The authors of this research paper indicate that the presented solution may be applied to both approaches.

Maréchaux (2006) has developed an approach based on event-driven architecture. This approach has been utilised to allow registration, revision and efficient handling of events with NC occurrence. The presumption is that the solution presented in this paper may be utilised for reporting, revision and realtime decision making.

Many modern manufacturing systems are equipped with adequate buffers so that the deadlocks in scheduling and production are reduced to a minimum. However, conditions that will produce a deadlock may occur in some emerging manufacturing industrial departments that are frequently supplied with an inadequate number of buffers and are even without buffers (Taiping et al. 2014). In these cases, reducing and avoiding deadlocks becomes a significant issue. The solution presented in this paper can be used to implement deadlock detection/recovery and avoid strategies for solving deadlock issue since it enables the necessary integration of software components such as statistical modules, communication modules and databases via web service technologies (Xu et al. 2016). Detection/recovery strategy utilises 
statistical and communication modules to identify deadlock event and ensure a mechanism to release the resources in the case of deadlock event.

Additionally, in the production scheduling strategy for detection/recovery of deadlocks with uncertain resources, reactive locally constituted real-time decisions are necessary (Lu et al. 2017), which can be achieved by applying the mentioned modules. Donauer et al. (2015) adapted and used KDD as a quality tool with the integrated Herfindahl-Hirschman Index as the core element. In the same paper, the results are presented in matrix-based patterns, which can be applied to recognise preventive/corrective measures patterns. The presumption is that NC application, proposed in this paper, may provide a recurrent neural network (RNN) module for pattern recognition and statistical tools such as Histograms, Pareto diagrams and Scatter diagrams.

Whether it is the production process, for which most QC solutions are designed, or the assembly process performed with parts procured from suppliers, the final product often does not meet customer specifications. Software solutions for NCs identification are mainly based on visual detection of NCs like machine vision proposed by Karathanassi et al. (1996), industrial photogrammetry (Bösemann 2005), and industrial computer vision (Villalba-Diez et al. 2019). Even today, most companies use experienced engineers to identify faults in products visually. QC based solutions are presented in Table 1.

As point out in Table 1, the general goals for the solution presented in this paper differ from other solutions in few categories: (1) to be equipped with barcode readers for ease of use if barcodes are implemented in the production process; (2) to be affordable and suitable for the automotive industry SME suppliers; (3) to be equipped with statistical and RNN modules and in compliance with most of the analytic modules.

In compliance with data stated in Závadská and Závadský (2018), related to the methods and techniques of 14.0 currently in use, the following Table 2 is defined for all those techniques used to manage NC.

Following the data obtained from Table 2, the authors have decided to use smartphones/tablets and barcode. Since these technologies are increasingly empowered, allowing a simple transition from the presentation to the business logic development, the following section will outline their benefits to create the proposed NCM solution.

\section{Architecture And Characteristics Of Developed Solution For Nonconformity Management}

\subsection{General software components design, their relationships and available technologies for Q4.0 consolidation}

The general software components design and their relationships are, in most cases, implemented in three tiers, i.e. presentation, application and database tier. The presentation tier implies user interface at the applications' top-most level, with the primary purpose to handle clients' requests and to present adequate 
and understandable results. Development trends primarily include applying JavaScript technologies, JavaScript frameworks, or alternative frameworks based on PHP, Java, and Ruby programming languages when implementing the Web browsers' presentation tier. In such circumstances, communication with other tiers is performed through API calls. The application tier is hosted on in-house servers or a distributed server in the cloud, depending on the overall software solution's processing power requirements. In the contemporary industry environment, cloud computing can play a premier role in the transformation process since it is a type of outsourcing technology that combines many scalable, normally personalised computing servers and inexpensive infrastructure (Wang et al. 2016). The data access tier provides entry to data located within the practical solution boundaries or data available through remote cloud service providers (Núñez et al. 2020).

Additionally, it is possible to easily change data storage technology, i.e. different database management systems, moving from one to the other, without significant influence on business logic. Dividing components with adaptable relationships into tiers yields a few advantages, including: overall modularity, making it easier to manage and maintain separated components, then to tackle the entire architecture; mutual independency between technologies and technology stacks used to incorporate various tiers; and separate back-end tier allowing to deploy different databases (Luís Pereira and Costa 2019), with the possibility to scale up, extend by adding multiple web servers, depending on the SMEs future growth, and, therefore, produce decentralised applications. With the stated facts in mind, it can be observed that a significant number of technologies in different layers can be used to implement the software solution.

\subsection{Incorporated application and introduction of advantages for Q4.0 and NCM}

In this section, the objective is to elaborate the general solution description introduced in Fig. 1 in order to comprehend the SMEs needs and to highlight the current trends of 14.0 (including centralised data platforms, a sense of urgent achievement of continuous improvements, and real-time communication channels), which are influencing Q4.0 among other concepts.

Based on the stated presumptions and intentions, the requirements for an NC reporting software solution are as follows: the solution has to be able to report stand-alone NC and to assign NC to a specific employee to be managed and resolved; to record one or more preventive/corrective actions and to associate them with reported NCs; to allocate responsibilities for the implementation of the preventive/corrective actions; to investigate notifications, with reminders, to determine correlations between process performances and NCs, within specific periods, utilising statistical analysis methods; to use data storage and further subsequent use to form the necessary knowledge in KDD for the future decision-making processes. Accordingly, the software solution contains the following modules: statistical and communication modules, the module for possible product barcode detection, the module for initial NC reporting, the module for pattern recognition, the module for statistical analysis graphical presentation. 
Having in mind that each enterprise has its procedures on dealing with NCs, it is possible to list general activities the software needs to provide: (1) Definition, containing a description of requirements that are not met according to demands specified by stakeholders, defined or undefined needs, needs of the organisation itself, customers expectation. (2) Identification of the product, with the barcode initial product details detection. Identifying the product is usually the first step in quality-related issues, especially in complex and dynamic processes with multi-factorial and non-linear interactions. (3) Management (when NC of the product accrued, who decided, status, location) of all kinds of issues from an individual or different locations gathered in one consolidated system. (4) Actions related to NCs (Rework, Repair, Permissions, Corrections, Scrap) enable organisations to archive and administer containment processes related to lots, units and large quantities systematically evaluated against the corrective, preventive action requirements. (5) Responsibilities, i.e. identifying quality practitioners responsible for NC removal. These actions are the recommendation of ISO 9001:2015. The solution can be included in the loT-architecture of the MOs and connected with other databases via web services. The collected data related to the definition NC, its management, identification of products or processes in which NC has occurred, and the proposed actions to resolve the NC can be used to form KDD that may be subsequently utilised, alongside RNN, for pattern recognition and proposal of appropriate preventive/corrective actions. Therefore, the solution features are based on Schmitt et al. (2020), so that it is:

- distributable, applicable from different mobile devices and easy to use for real-time NC digitalised reporting,

- general, independent from the mobile device platform,

- adaptable, so that the effect of an increased number of reports and users can easily be overcome,

- modular, changes in one part of the solution, do not affect the rest of the solution, and

- inexpensive, independent from the industry type.

Based on the solution stack's virtualisation and management, cloud computing solutions can be divided into three different levels, including infrastructure, platform or Software as a Service (SaaS). This software's main idea is to be used as SaaS, starting from the ground requirements to be distributable, general, adaptable, modular, and consequently scalable, the increasing number of users will not represent any problem. Therefore, Fig. 2 shows the implementation of the proposed solution based on JavaScript MEAN frameworks' application. The expression MEAN stack represents a set of JavaScript-based technologies appropriate for web applications development. MEAN is an abbreviation derived from the first letters of the used technologies, i.e., MongoDB, ExpressJS, Angular and Node.js. MEAN is an opensource stack with the possibility to develop adjustable and scalable applications, and as such, it is the perfect candidate for cloud hosting (IBM cite 2020). The presentation tier is developed through the Angular framework; the application tier is introduced through Express.js and Node.js capabilities, whereby the MongoDB was adopted to define the cloud database. Consequently, technology selection can be considered as an essential aspect, as it affects the cost, performance and possible functionalities of the NCM reporting solution and the overall Q4.0. 
If multiple companies apply the solution, collected and integrated data could be used for pattern recognition. In this case, they will be able to learn from each other and have patterns for recognition of $\mathrm{NCs}$ and the selection of possible management initiatives.

The proposed method has clear advantages because it utilises a new approach in application development to fulfil the principles of QMS. This approach with availability and personalisation increases employees' participation and involvement and provides a broader base for NCM and evidence-based decision making.

The presentation tier is deployed through JavaScript/TypeScript[1] open-source Angular framework. Increased use of Angular framework is derived from the fact that it supports Progressive Web Applications' concept. This concept indicates that an introduced application is: instantly loaded, independent from the network slow or unstable connection, responsive, and, among other things, with features, such as Web Bluetooth[2] API, that makes the application to be more native-like. Node.js represents an asynchronous open-source JavaScript run-time environment carried over Google Chrome's JavaScript engine V8[3], to create scalable applications independent from the browser. Besides the V8 engine, Node.js contains an abstraction layer libuv[4] library to handle asynchronous events. Node.js unifies application development merely around JavaScript programming language[5]. The NC reporting data are sent from the presentation layer to the business layer in a lightweight data-interchangeably JavaScript Object Notation (JSON) format. This format is language-independent, and it has been fitted for data sharing among interconnected clients. As compact and straightforward, JSON is efficiently generated and parsed by machines and easily read by humans. Based on the mentioned advantages, JSON turned out to be a fitting solution for cloud NoSQL database within this study.

Additionally, the strategy of mitigating deadlock may be conducted dynamically by utilising communication modules and MongoDB NoSQL database to acquire up-to-date workflow information and control the allocation of resources. MongoDB databases belong to the NoSQL databases class, unlike SQL relational databases, where columns are eliminated, and rows are documents used to store information about the data and the data itself. Since the data are stored in a BSON format that presents a binary JSON document, MongoDB proved to be a reasonable solution for JavaScript-centric application development. This module adds an entire tier of features on top of MongoDB that enables definition and maintenance of data structure and data models and their utilisation so that direct interaction from application code to the database is performed.

To sum up, direct communication with the MongoDB database can only be done through the Mongoose module, which further communicates with the Express and Node.js. In turn, Angular can not communicate to mongoose; it only communicates directly with Express. MongoDB enables parallel data displaying but does not allow parallel data entering nor simultaneous parallel data displaying/entering. The authors of this paper avoided a possible deadlock by defining a maximum number of data entry attempts and automatically discharging each operating lock with the request termination, in that manner allowing a process to be sophisticatedly terminated. In contrast, the other process completed its task. Furthermore, 
MongoDB provides some convenient metric parameters obtained through the db.serverStatus() command to determine the locking influence on database performance. In reporting and resolving the NC issues, the authors of this paper analysed and resolved how to arbitrate shared resources and implement process synchronisation.

\subsection{Work plan of the digitalised nonconformity management and usage of the system}

The graphical illustration of workflow, emphasising the detection of NCs, is presented in Fig. 3. The process of NCM starts with an NC detection (activity 1.). Looking at an arbitrary enterprise, the authors assumed that the detection of NCs is under the responsibility of each employee of the organisation. According to ISO 9001 requirements, employees are empowered and responsible for identifying, within their competence and expertise, an NC in the processes they participate in. Each enterprise has to provide a representative for NCM (Quality engineer). After that detection of NC, in this example, an employee or a quality engineer scans the barcode with the assistance of the application described in this paper, creates and forwards a report consisting of product identification, documentation, and disposition of NC to cloud server (activity 2).

Cloud solution could encompass adaptive business intelligence tools (mentioned earlier in Sect. 1 of this paper, e.g. tools for root cause analysis) to detect NC occurrence patterns and send a notification to the quality engineer about the severity of NC with proposed preventive/corrective measures. In this way, the emphasis may be placed on all employees' evidence-based decision-making and involvement (activity 34).

After the NC is solved, the quality engineer sends feedback to the developed solution: a) notifies the process owner about the NC status, and b) integrates new data into the existing database, available for further RNN training and application (activity 5-6).

Automation of NC reporting and proactive contribution of all employees represents an organisation's significant achievement to foster quality culture and reach quality excellence. The long-term benefit of the digitalised NC reporting is to enable in-depth analysis of production performances by employing adaptive business intelligence tools.

\subsubsection{Software solution capabilities}

The software solution aims to provide real-time reporting in the NC occurrence caused by deviations in organisation or supplier processes. Adequate NC reporting should comprehend critical elements, such as positioning, identification, documentation, and disposition. A detailed description of these critical elements is presented below.

1. The positioning of $\mathrm{NC}$ occurrence in $\mathrm{MO}$ through the application graphical user interface (GUI) with the selection from the provided predefined list of organisation locations. Other possibilities include 
the application of API maps with interactive geocoding of NC location occurrence. However, within this application, the mentioned option is avoided since it is presumed that the production workspace is predefined, and the application of most of the API maps demands additional cost.

2. Identification enables organisation employees, with the option to apply application incorporated barcode reader and to gather initial corresponding product data, stored in the centralised database associated with a specific barcode when related NC issue occurs. A number of the initially predefined product data may differ, depending on the organisations' type (i.e. industry type, organisation size, type of activities, available technology, practicality/feasibility, resources).

3. Documentation of NC provides employees with the possibility to describe and document detected NC issue. The NC description involves text note and details, including product amount, work order, series and operations number, to document the NC issue properly.

4. Disposition includes the possibility to select one or more different options from a predefined list of options to resolve the perceived NC issue. This initial subjective assumption to resolve the NC issue is obtained by the employee. Accordingly, the NC issue has to be assigned and evaluated by the process owners (responsible for maintaining product quality at an adequate level). Besides information regarding NC disposition, this element of the application provides statistical data for an organisation, enabling the estimation of possible proactive measures for future cases.

The solution can get the data generally from three sources:

1. The first source is manual input from employees (it enables an employee to make the NC report and send it to the central database and even get a suggested solution).

2. Employees could use a mobile phone as an instrument with some basic measuring options. Generally observed, through the Android diagnostic framework, an extensive spectrum of sensors for measuring different parameters is supported (motion detection and rotation detection, monitoring air temperatures, rotation detection, air pressure changes, monitoring dew point, absolute, and relative humidity) (Android - Sensors Overview 2020). Android provides support for different sensors, which could be divided into two categories: a) hardware-based - they derive data by directly determining the value of the specific product and process parameters and production conditions, such as temperature, air quality, or geolocation; b) software-based (virtual sensors or synthetic sensors) they are not physical devices, but they are mimic hardware-based sensors. For the proposed application, the authors of this paper used the sensors mentioned above, all available at Android 4.0 API Level 14 (most of them were also present in Android 2.3 API Level 9, excluding temperature and humidity sensors).

3. The application can utilise the data gathered directly with the sensors incorporated in production and IoT. Through these sensors, dedicated software incorporate functionalities of external devices or sensors connected to peripheral $1 / 0$. This option demands customising the solution on the companies perimeter, which includes existing sensors in the system.

In addition to the NC issue reporting's critical features, the solution has a communication channel that enables the circulation of timely information between employees, quality engineers, and process owners. 
The task assignment and adequate communication are performed in the form of direct messaging and $\mathrm{NC}$ identification cards interchange, with the process owners' ability to initiate the creation and completion of NC identification cards. The initial exchange of information commences when the report is generated within the database, and the process owner receives a message on receipt of the report. The message includes aggregated data (position, documentation and options to resolve NC issue), based on which process owner determines the dynamics and defines employees tasks to solve problems and identify root causes. The assigned task contains NC information, accompanied by an initial message derived from the process owner and any messages that follow the overall NC resolving process stream. Employees can review their individual previously entered KDD data, while process owners may review particular NC resolving states and overall NC issues. Lastly, within the presented solution, it is possible to utilise the Recurrent Neural Network (RNN) to detect possible patterns for preventive/corrective measures (Lukoševičius 2009). RNN is a neural network (NN) class that extends the conventional feedforward NN with loops in connections (Mou et al. 2017). RNNs differ from feedforward NN since they can process sequential input data through a recurrent hidden state whose activation in each subsequent step depends on the previous step's activation. In this way, the RNN can expose dynamic behaviour.

For the NC and corrective actions pattern recognition, the given sequence is $n c=\left(n c^{1}, n c^{2}, \ldots, n c^{k}\right)$ representing NC occurrence descriptions so that the RNN framework can calculate the hidden vector sequence as $h=$ $\left(h^{1}, h^{2}, \ldots, h^{K}\right)$ by iterating the following equation from $\mathrm{k}=1$ to $\mathrm{K}$ :

$$
h^{k}=\phi\left(w_{i h} n c^{k}+W_{h h} h^{k-1}+b_{h}\right)
$$

Where $w_{i h}$ indicates the input-hidden weight vector, while $W_{h h}$ denotes the context weight matrix of the hidden layer, $b_{h}$ is the bias vector in the hidden layer, and $\varphi 0$ is the hidden layer activation function (possibly sigmoid or tansigmoid function). Finally, predicted corrective action $q_{a}$ could be computed as follows:

$$
q_{a}=W_{o h} h^{K}+b_{o}
$$

Where $W_{o h}$ indicates the output-hidden weight matrix, and $b_{0}$ is the bias vector of the output layer. The motivation is to present different $\mathrm{NC}$ occurrences and corrective actions as sequential data so that an RNN can be adopted to model pattern recognition.

\subsubsection{JavaScript MEAN stack approach for real-time nonconformity management in SMEs}

In this section of the paper, the software solution functionalities for common SME have been demonstrated. The assumption is that the SME has up to 250 employees so that employees and process owner perform the QC. Therefore, the process owner takes the role of the personnel in charge of NCM. The general objectives of this software solution are a) NCM digitalisation, b) overall time of information transmission reduction and c) facilitated communication.

\subsubsection{NC reporting software application process flow and properties}


The proposed solution's process flow is introduced in Fig. 3, while its properties for both employee and process owner roles are presented in Fig. 4 and Fig. 5. System utilisation begins with the initial registration and logging on to the system each time the system is used. Both types of roles have somewhat similar functionalities, including (1) NC reporting (Report property introduced in Fig. 4, with a detailed description in the previous section), (2) unresolved NC occurrences (Current cases property in Fig. 5), and (3) statistical NC data, regarding NC solving results (Solved cases stats property in Fig. 5), (4) communication channel (Communication property in Fig. 5).

The property "Current cases" presents all unresolved NC occurrences that are delegated to the logged-in user. Hence, it is possible to select one of the unresolved cases and determine the activities that need to be taken to resolve NC in real-time (Fig. 5, section "List of open nonconformities"). Furthermore, intending to find the adequate procedure for current NCs occurrences to be resolved, it is possible to review the statistical data from the previous period stored in the cloud database (Fig. 5). The NC solution can be defined in collaboration with other employees that are authorised and subscribed to a specific predefined communication channel so that they may receive and trigger messages in real-time, more like social networks (Fig. 5, input filed "Message" and previous messages exchanged through a communication channel).

As already mentioned, the functionalities of the two application parts differ based on the defined roles. Process owners part encompasses a broader insight: the ability to review all NCs at the process level (overall unresolved NC occurrences, overall statistics, employees that reported NCs), unlike employees' section that can only be used to review NCs, entered by a specific employee.

\subsection{CASE STUDY AND INITIAL RESULTS OF IMPLEMENTATION}

This section intends to demonstrate two things: the first is to demonstrate the system's usage on the reallife example (example: the surface scratch of plastic product detection), and the second one to present data from this system utilisation in three SMEs.

The developed solution has been used in three SMEs in plastic and rubber parts production intended for the automotive industry. The NCs of plastic and rubber products could be different, hard to determine and report, or significantly different to detect using classical control systems.

The following real-life example depicts the advantages of the developed system. Figures 6 and 7 depict the utilisation of software solution for surface scratch of plastic product detection. These NCs could emerge for different reasons. By applying the solution, the employee can document and report NC. After analysis, decision-makers defined the corrective procedure and decided that scratches larger than $3 \mathrm{~mm}$ represent the product's defect.

The next time when an employee detects scratch/NC, it is possible to provide measurement or use correlated barcode data to trace previous similar product of NCs, to acquire the knowledge necessary to 
presume what to do with this type of NC. If a scratch is less than $3 \mathrm{~mm}$, the product will go further in the production process; otherwise, the defective products will be excluded from further processing. The software system using the RNN module provides the mentioned decision and action.

In the second step, the authors of this paper monitored the implementation and initial results of represented software in three SMEs. The data were gathered for six months. Having in mind that companies have different production programs and amounts of production and different products, it could be concluded that implementing and utilising such a system improves employees' involvement in the process and quality culture. On the other hand, it enables easier detection, reporting and digitalisation of spotted NCs and reduces reporting and taking actions periods.

All companies have introduced, implemented and certificated QMS according to ISO 9001:2015. The process approach enables the organisations to control the interrelationships and interdependencies among the system processes to enhance the overall organisation performance. The process approach utilisation in a QMS empowers: a) user perspective inclusion and consistency when meeting user demands; $b$ ) the examination of the activities in the sense of added value; $c$ ) the accomplishment of effective process performance; d) enhancement of processes based on evaluation of data and information (ISO 9001:2015). According to these reasons, the pre-request, that all companies where software system is tested, have implemented ISO 9001, ensured the same principles and same starting point for all three cases.

The solution provides statistical data from previous examples and improves the process of QC and NC assessment, recommendations and measures taken (Table).

Data presented in Table 3 introduced some indicators for the measurement of NCs and provided an opportunity to compare and correlate different periods and different companies. These indicators could help benchmark different solutions and enterprises. Finally, the authors detected that recognising the patterns using RNN could help take managerial actions in NCM and QM. From the obtained and presented data, it is evident that the number of detected and reported NC and the number of corrective and preventive actions taken have been increased. It is essential to consider that many measures taken were based on suggestion developed on pattern recognition. Besides, this user-friendly software, with innovative modules, improves employees' participation in NC reporting. It may be concluded that this solution enables important principles of QM: participation of employees and evidence-based decision making for quality managers.

In the second place, the authors of this paper presented data where the correlation between NCs and sections of ISO 9001:2015 standards were established (Table 4). This correlation table allows quality managers to track back all NCs and have clear managerial implications for future inner and external audits.

Applying the software makes it possible to connect NCs with specific sections of the ISO 9001:2015 standard. Each company could have evidence of their data and define managerial actions according to 
the detected NCs primary sources. According to Table 4, it could be observed that most NCs could be connected with Sect. 8.7 and 8.5, but also significant NC number is related to 6.2.

Using this information, quality managers could work on the improvement of all specific processes and the improvement of the quality system as a whole. Also, companies could work together and share their databases and patterns to benefit mutually and contribute to an extensive base for the system to learn.

To further extend the case study and consider the developed solution's performance, tests were performed to compare the developed solution with the possible solutions based on the affordable technologies including PHP, Apache server, MySQL and MariaDB. The authors tested the solutions on the identical infrastructures, which included servers for data storage with structures based on technologies MySQL/Apache/PHP, MariaDB/Apache/PHP, and MongoDB/Node.js/Express.js, and remote users presentation tiers with structures based on an application of HTML/CSS, HTML/CSS, and Angular, respectively. Testings reflected the most realistic possible scenarios to determine which technologies best performance in terms of forwarding queries and data retrieval from the databases.

Different NCs query workloads were created to test the previously used solutions' databases and current solution responsiveness. Each benchmark test was performed under the same quality manager user credentials, applying the default database settings.

First of all, there were three scenarios, including data insertion, modification, and selection. The first scenario is used to test the insertion agility for a more extensive set of data objects in a specific request, the second scenario to test the modification agility and the third scenario to test selection agility. Each test scenario was performed five times for different query quantities so that a mean value was calculated for each test type. The procedure was repeated due to variations that may occur during the execution of the tests. The tests contained syntax and structure, as shown in Table 5.

The tests introduced in this part of the study have been performed on a single server, but things might look different with data shared across clusters. This fact should be taken into account in future testing. Tables 6, 7, and 8 clearly show that the solution based on MongoDB has higher velocity when it comes to data insertion, modification, and retrieval compared to the solutions based on MySQL and MariaDB, mainly when dealt with a considerable amount of data. The increase in time for all three types of solutions DBMS seems to be linear. In the scenarios and circumstances covered in this study, the authors discovered that by switching from MySQL and MariaDB technologies to MongoDB technology, it is possible to get a significantly faster database with a relatively similar structure.

\section{Discussion}

\subsection{The relevance of real-time reporting of NCs through edge devices}

As modern technologies can dynamically scale, with advantages that include high availability and maintainability, constant data accessibility and functionalities from each connected edge device, it is 
essential to emphasise that organisations must not overlook the potential of 14.0 solutions and services. Considering the nature of 14.0 technologies to change and evolve continually, there are numerous application possibilities and benefits. Therefore, an increasing number of organisations from different business areas are intensively incorporating these advanced technologies to improve their business capabilities and capacities at a minimum cost (Zhong et al., 2017). MO can utilise service-oriented architecture via the Internet to acquire customisable, flexible, collaborative, and reconfigurable services to their employees, decision-makers and customers, with the consequence of providing a powerfully unified human-machine manufacturing system (Feeney et al. 2015).

Based on the facts presented, SMEs are advised to broaden current inhouse, production-oriented applications to improve 14.0 solutions (Birkel et al. 2019; Da Silva et al. 2020). Additionally, the capacity to audit quality comprehensively and accurately is a fundamental achievement factor for manufacturing companies in today's global market. Thus, 14.0 solutions in the field of quality can provide beneficial inspection strategies. The idea is implemented in the shape of an NC software solution and demonstrated for a case study in the automotive industry. The results of the software solution application contribute to the embracement of the QMS principles. Primarily, according to the presented results, the NC solution is focused on the process approach, leadership, evidence-based decision making, improvement, and relationship with suppliers. The step towards the realisation of the process approach principle is reflected in the possibility of applying the software in each process individually so that specific processes have specific NCs and correlated preventive and corrective actions KDD. NC solution promotes leadership since it creates conditions in which people are inspired, encouraged and recognised for their contribution to quality achievement. NC solution can provide data and information to the relevant people, and it may ensure that these data and information are precise and reliable for evidence-based decision-making. Pattern recognition models are globally trained based on the knowledge acquired from the cloud data repository regarding NCs and corrective actions and locally exploited on mobile devices, fostering continual improvement. Throughout the MO processes, NCs are identified and reported through the edge devices, on which data handling and model application are processed in near real-time. The predicted corrective actions are evaluated and possibly incorporated at the process level, providing progressive inspection decision-making. Depending on the process level results, reporting time and additional time for the definition of actions taken can be shortened, generating an interesting business concept. Thus, prompt gathering and sharing of reliable information regarding NC with relevant providers can improve partnership relationships and enhance future deliveries in accordance with the requirements.

The amount of real-time data accumulated from multiple sources in production organisations is steadily increasing. If this data is stored, there is a tremendous opportunity to facilitate information flow and improve production processes with big data analytics and the right decisions (Zhang et al. 2017). To do so, conventional industrial organisations should incorporate the concept of 14.0 with main pillars, including internet technologies, loT, cloud computing, big data, and adaptive artificial intelligence (Tao et al. 2018). Therefore, this study's core premise is that one of the possible fundamental steps towards the 14.0, and subsequently, Q4.0 concepts incorporation, is to deploy mobile applications, able to enhance QM and production output. Accordingly, this study proposes a specialised role-based mobile application, with 
multiple interfaces divided by roles that can be utilised to report real-time NCs and to gain data-driven insight for subsequent production defect and root cause detection, defect mapping, machine failure and downtime reduction following Salkin et al., (2018) assumptions. Some of the benefits that the application brings with it are greater accessibility, collaboration and data transparency. The possible lack of the application regarding user data security in the cloud has been overcome since Node.js authorisation and authentication (i.e. bcryptjs, jsonwebtoken) modules were applied.

To sum up, the paper provides an observation regarding the potential contributions of 14.0 based solutions in QM. However, additional research is compulsory to scale the application of pattern recognition model-based inspection and confront emerging challenges and research questions. Future research could expand the conclusions, for instance, by investigating and implementing NC solution in a more significant number of organisations from different industry sectors. Additionally, extension in the number of specific process parameters and data sources can, in turn, lead to an expansion and increase in complexity of the solution. Nonetheless, complexity may also enhance the solution performance and increase NC reporting coverage by including a higher number of distinct NC and corrective action patterns.

It can be concluded that, through the increased data availability in MOs and the 14.0 technological advances in computing, the solution for NCs quality identification, reporting and pattern recognition is a promising approach to conduct quality inspection processes more economically.

\subsection{Justification of the overall software implementation and directions for further improvement}

After presenting the benefits of the software solution from a methodological (Sect. 3.2) and QM (Sect. 4.1) viewing angles, it is mandatory to highlight the benefits that the solution delivers in terms of cost-effectiveness, horizontal scalability, cloud compatibility, and overall adaptability. As discussed in Sect. 3.2, the proposed dynamic solution, commencing from the presentation tier to the database tier, is constructed by applying JavaScript free and open-source MEAN comprehensive stack. MEAN stack is less costly since it is necessary to invest time to master only one programming language than other technologies, which requires the knowledge of at least two programming languages to establish the entire software solution.

The novelty is in the presented modular software solution for NCs identification and reporting, that integrates the requirements of ISO 9001:2015 standard when NCs are reported, statistical and communication modules that can be used through end-to-end JavaScript environment and 14.0 technologies to create an environment for the Q4.0 concept establishment.

This paper is constituted on the 14.0 research starting from quality objectives to achieve conformity related to specifications, variations reduction, waste reduction, defects prevention, alignment between strategy and operation enhancement, efficiency and effectiveness of equipment and operations enhancement. 
Quality objectives are fulfilled by improving: connectedness with real-time reports of emerging insights; knowledge based on the utilisation of appropriate neural network method; and automation based on problem reporting and solving by an intelligent agent using mobile phones.

A fundamental novelty, which is presented in this paper, refers to the integration of research in QC, management, and software engineering. The developed software solution is intended to be used for affordable identification and reporting of $\mathrm{NC}$ with the possibility to integrate other software modules for quality analysis, problem-solving, corrective/preventive actions, and using a high level of applied methods (machine learning and artificial intelligence optimisation methods).

From the industrial perspective, the authors of this paper believe that our solution is interesting for technology providers aiming to release solutions for SMEs. The authors of this paper presented scalable, flexible, and, above all, affordable solutions form SMEs. SMEs usually do not have the financial resources to invest in large scale and expensive solutions. The presented solution is suitable for SMEs, but it is flexible and open and could be integrated with legacy systems and third-party solutions. Similarly to the 'e-ProMan' system (Katchasuwanmanee et al. 2016), the solution presented in this paper is primally designed to suit SMEs needs with strict budget limitations and lower data-gathering infrastructure resources. This paper has tried to present a solution that incorporates some of the vital e-manufacturing elements (Cheng et al. 2008). These elements include infrastructure for information sharing; a protected and security application; managed, shared information and knowledge; information to support management decisions; documented process support; electronically supported processes; and effective teamwork.

The real-time nonconformity management solution possibilities are primarily based on applying the sensors network for data collecting and a prediction module. By utilising the sensors' network, data are retrieved directly from the SMEs manufacturing lines, providing production parameter measurement and evidence of possible production NCs. Besides, some basic measures could be retrieved using mobile devices. Consequently, the RNN (recurrent neural network) prediction module for proactive/corrective actions selection may be applied to support real-time decision making. The main idea is that based on the data set on NCs that have previously occurred and the proactive/corrective actions applied to solve them, the module defines a list of proactive/corrective actions proposals for ongoing NCs. In addition to defined proposed actions, the list may assign a success rate to each action based on previously resolved similar cases. The most appropriate proposal adopted and possibly refined by the quality managers can then be applied in real-time and added to the database.

In compliance with the 14.0 paradigm, in the proposed software solution, modules for Big Data Analytics related to NCs and prediction of business process' state could be implemented. In this way, it will be possible to choose an optimal solution for problem-solving at the level of each machine, machine line, or enterprise as a whole.

In addition, this paper indicates that small, affordable solutions developed to be users friendly could incorporate some of the main principles of QM, such as employee engagement. In addition, the use of the 
MEAN stack provides an improved possibility for pattern recognition and fulfilment of other principles of QM, such as improvement and evidence-based decision making.

\subsection{Conclusion}

Achieving higher quality and meeting customer demands represents a fundamental goal of nowadays industries. This objective could be obtained by introducing an 14.0 based QC, which emphasises continuous monitoring, controlling, and improving organisational processes and products.

As NCM represents one of the essential tasks of QM, it is necessary to acquire and use ICT to facilitate information and enable the implementation of effective proactive and corrective QM measures in realtime. Along with identification, documentation of NC has emerged as a problem. Solutions on this topic from broad literature are mainly based on traditional measures and procedures for solving NC and are usually being taken only by QM representatives and require a significant amount of time. Some of these dilemmas could be untangled by the convergence between Quality, 14.0, and loT. Innovative software solutions could contribute to more advanced NCM.

The authors of this paper proved that the implementation and usage of affordable but advanced systems based on mobile platforms could improve the number of indicators connected with NCM. In addition to selected indicators, the authors presented the distribution of NCs according to sections in ISO 9001:2015 standard. Since the authors of this paper implemented a module to detect possible corrective actions patterns, the authors measured and presented several corrective actions taken based on defined patterns. The authors also believe that no single research presented such results and solutions so that modern NCM could benefit from the proposed approach. The use of technologies from the 14.0 pack could contribute to better and more effective NCM and QM generally by tracing the path to Q4.0 and QMS 4.0. Consequently, the use of new solutions and technologies could improve even basic principles of QMS (such as involvement of employees and innovation).

The presented software included some demands and unique features that differ it from similar solutions, primarily in the module where the: 1) Android Sensor Framework employed sensors from mobile platforms, that also the authors of this paper used; 2) novel RNN model could effectively analyse possible NCs, as sequential data determined corrective action, based on the knowledge about previously recognised NCs; and 3) authors enabled mobile platform to collect data from other sensors and loT. It ensures that this solution surpasses similar products, bringing additional value to organisations. The presented solution enabled identifying products or resources, turning the mobile platform into a barcode reader, enabling full reporting traceability. When NC product or situation accrued, established algorithm forwarded task to dedicated quality manager suggesting possible actions. The authors implemented a module for Recurrent Neural Network (RNN) to detect possible patterns for corrective actions. This module will enable the responsible person to use some defined patterns or make a decision using suggestion or making entirely new actions. Besides, the solution could help define the same actions, such as rework, repair, permissions, corrections, and scrap. 
The software solution brings benefits for both employees, managers and stakeholders through (1) involvement of all employees; (2) digitalisation and improvement of existing NC reporting systems; (3) improvement of NC culture; (4) better understanding and measurement of employer contribution to NCM; (5) making the technology ease of use both affordable and suitable for broader industry audience (the source code of a demo version, is publicly available on the GitHub repository: https://github.com/cqmquality-center/nonconformity.

Finally, the developed solution is implemented in 3 SMEs, and initial results show several benefits in increasing the number of NC detection, reporting, time, and increasing participation of employees number of preventive and corrective actions and improved QM system as a whole. The number of corrective and preventive actions was taken based on the assistance of the RNN module. All NCs were related to specific sections of ISO 9001:2015 standards so that quality managers and managers in the companies could have insight into the sources of the issues and the foundation for defining different managerial actions.

It could be concluded that 14.0 has been making a significant impact on all fields, including QM and NCM. QM could benefit by using the technologies from 14.0 tools, such as loT, cloud computing, artificial intelligence and cloud computing and modern ICT solution. In this paper, the authors suggested approach, presented, and tested software solutions that proved that the number of benefits could be achieved using innovative software systems and technologies. This paper also indicated the direction for the development of affordable open source solutions where the authors of this paper enabled SMEs to have benefits that were reserved, until now, only for large companies that have been using expensive software platforms. Besides, user-friendly software solution promotes employees' participation and the fulfilment of other QM principles.

The system could be upgraded and expanded in many different directions, such as development modules for documentation management or advanced system image recognition (as part of QC) to support and interconnection with other systems (safety, environment management).

\section{Nomenclature}




\begin{tabular}{|c|c|}
\hline Nomenclature & Referred to \\
\hline Al & Artificial Intelligence \\
\hline API & Application Programming Interface \\
\hline BSON & Binary JavaScript Object Notation \\
\hline CPS & Cyber-Physical System \\
\hline GUI & Graphical User Interface \\
\hline 14.0 & Industry 4.0 \\
\hline ICT & Information Communication Technology \\
\hline IloT & Industrial Internet of Things \\
\hline loT & Internet of Things \\
\hline ISO & International Organization for Standardisation \\
\hline IT & Information Technology \\
\hline JSON & JavaScript Object Notation \\
\hline KDD & Knowledge Discovery in Databases \\
\hline MO & Manufacturing Organisation \\
\hline NC & Nonconformity \\
\hline $\mathrm{NCM}$ & Nonconformity Management \\
\hline NN & Neural Network \\
\hline Q4.0 & Quality 4.0 \\
\hline QC & Quality Control \\
\hline QM & Quality Management \\
\hline RNN & Recurrent Neural Network \\
\hline SaaS & Software as a Service \\
\hline SMEs & Small and Medium Enterprises \\
\hline
\end{tabular}

\section{Declarations}

- Ethical Approval The authors declare that they have no conflict of interest.

- Consent to Participate The authors declare that they have voluntarily agreed to participate in this research study 
- Consent to Publish The authors consent to the publication of the manuscript in The International Journal of Advanced Manufacturing Technology, should the article be accepted by the Editor-in-chief upon completion of the refereeing process.

- Authors Contributions A.Đ., MS and TC conceived of the presented idea. A.Đ. developed the theory and performed the computations. ME and MM verified the software solution modelling and appication. MS investigate Quality 4.0 concept and supervised the findings of this work. All authors discussed the results and contributed to the final manuscript.

- Funding This research received no specific grant from any funding agency in the public, commercial, or not-for-profit sectors.

- Competing Interests The author declare that they have no significant competing financial, professional, or personal interests that might have influenced the performance or presentation of the work described in this manuscript.

- Availability of data and materials The authors declare that software solution code is available at the GitHub repository: https://github.com/cqm-quality-center/nonconformity

\section{References}

1. Alcácer V, Cruz-Machado V (2019) Scanning industry 4.0: A literature review on technologies for manufacturing systems. Engineering Science Technology an International Journal 22(3):899-919

2. Android - Sensors Overview (2020) Retrieved 30 October 2020, from https://developer.android.com/guide/topics/sensors/sensors_overview

3. Barton D, Court D (2012) Making advanced analytics work for you. Harvard Bus Rev 90(10):78-83

4. Birkel HS, Veile JW, Müller JM, Hartmann E, Voigt KI (2019) Development of a risk framework for Industry 4.0 in the context of sustainability for established manufacturers. Sustainability 11(2):384

5. Bloom N, Garicano L, Sadun R, Van Reenen J (2014) The distinct effects of information technology and communication technology on firm organisation. Manage Sci 60(12):2859-2885

6. Bonilla SH, Silva HR, Terra da Silva M, Franco Gonçalves R, Sacomano JB (2018) Industry 4.0 and sustainability implications: A scenario-based analysis of the impacts and challenges. Sustainability 10(10):3740

7. Bösemann W (2005) Advances in photogrammetric measurement solutions. Comput Ind 56(89):886-893

8. Cheng K, Bateman RJ (2008) e-Manufacturing: Characteristics, applications and potentials. Prog Nat Sci 18(11):1323-1328

9. Da Silva VL, Kovaleski JL, Pagani RN, Silva JDM, Corsi A (2020) Implementation of Industry 4.0 concept in companies: Empirical evidences. Int J Comput Integr Manuf 33(4):325-342

10. Djekic I, Tomasevic I, Radovanovic R (2011) Quality and food safety issues revealed in certified food companies in three Western Balkans countries. Food Control 22(11):1736-1741 
11. Donauer $\mathrm{M}, \mathrm{Peças} \mathrm{P}, \mathrm{Azevedo} A$ (2015) Identifying nonconformity root causes using applied knowledge discovery. Robotics Computer-Integrated Manufacturing 36:84-92

12. Farooq MU, Waseem M, Mazhar S, Khairi A, Kamal T (2015) A review on Internet of things (IoT). International Journal of Computer Applications 113(1):1-7

13. Feeney AB, Frechette SP, Srinivasan V (2015) A portrait of an ISO STEP tolerancing standard as an enabler of smart manufacturing systems. Journal of Computing and Information Science in Engineering, 15(2)

14. Foidl H, Felderer M (2015, November) Research challenges of industry 4.0 for quality management. In International Conference on Enterprise Resource Planning Systems (pp. 121-137). Springer, Cham

15. Geissbauer R, Vedso J, Schrauf S (2016) Industry 4.0: Building the digital enterprise. Retrieved from PwC Website: https://www. pwc. com/gx/en/industries/industries-4.0/landing-page/industry-4.0building-your-digital-enterprise-april-2016. pdf

16. Gilchrist A (2016) Industry 4.0: the industrial Internet of things. Apress

17. Giorgetti A, Cavallini C, Ciappi A, Arcidiacono G, Citti P (2017) A holistic model for the proactive reduction of nonconformities within new industrial technologies. International Journal of Mechanical Engineering Robotics Research 6(4):313-317

18. Gunes V, Peter S, Givargis T, Vahid F (2014) A survey on concepts, applications, and challenges in cyber-physical systems. KSII Transactions on Internet \& Information Systems, 8(12)

19. Hao Y, Helo P (2017) The role of wearable devices in meeting the needs of cloud manufacturing: A case study. Robotics Computer-Integrated Manufacturing 45:168-179

20. Hashim J (2015) Information communication technology (ICT) adoption among SME owners in Malaysia. International Journal of Business and Information, 2(2)

21. Hermann M, Pentek T, Otto B (2016, January) Design principles for industrie 4.0 scenarios. In 2016 49th Hawaii international conference on system sciences (HICSS) (pp. 3928-3937). IEEE

22. Huang B, Li C, Yin C, Zhao X (2013) Cloud manufacturing service platform for small-and mediumsized enterprises. The International Journal of Advanced Manufacturing Technology 65(912):1261-1272

23. International Organization for Standardization (2015) ISO 9001:2015 quality management systems requirements, Geneva

24. ISO (2018) The ISO Survey. [online] Available at: https://www.iso.org/the-iso-survey.html [Accessed 9 Dec. 2019]

25. Ivanov N (2016) Analysis of the data using a semantic network as a tool for management nonconformities in quality management system. Modern Applied Science 10(1):47

26. Jacob D (2017) Quality 4.0 impact and strategy handbook: getting digitally connected to transform quality management. LNS Research, Cambridge

27. Jia L, Chen C, Liang J, Hou Z (2017) Fabric defect inspection based on lattice segmentation and Gabor filtering. Neurocomputing 238:84-102 
28. Karathanassi V, lossifidis C, Rokos D (1996) Application of machine vision techniques in the quality control of pharmaceutical solutions. Computers in industry 32(2):169-179

29. Katchasuwanmanee K, Bateman R, Cheng K (2016) Development of the Energy-smart Production Management system (e-ProMan): A Big Data driven approach, analysis and optimisation. Proceedings of the Institution of Mechanical Engineers, Part B: Journal of Engineering Manufacture, 230(5), 972-978

30. Kim H, Jung WK, Choi IG, Ahn SH (2019) A Low-Cost Vision-Based Monitoring of Computer Numerical Control (CNC) Machine Tools for Small and Medium-Sized Enterprises (SMEs). Sensors 19(20):4506

31. Kim J, Abdelzaher T, Sha L, Bar-Noy A, Hobbs R, Dron W (2016) On maximising quality of information for the Internet of Things: A real-time scheduling perspective. Embedded and Real-Time Computing Systems and Applications (RTCSA), p. 201- 211

32. Koh L, Orzes G, Jia FJ (2019) The fourth industrial revolution (Industry 4.0): technologies disruption on operations and supply chain management. International Journal of Operations \& Production Management

33. Kolberg D, Zühlke D (2015) Lean automation enabled by industry 4.0 technologies. IFACPapersOnLine 48(3):1870-1875

34. Lampropoulos G, Siakas K, Anastasiadis T (2018) Internet of Things (IoT) in Industry: Contemporary Application Domains, Innovative Technologies and Intelligent Manufacturing. people, 6, 7

35. Li X, Li D, Wan J, Vasilakos AV, Lai CF, Wang S (2017) A review of industrial wireless networks in the context of Industry 4.0. Wireless networks 23(1):23-41

36. Liao Y, Deschamps F, Loures EDFR, Ramos LFP (2017) Past, present and future of Industry 4.0-a systematic literature review and research agenda proposal. International journal of production research 55(12):3609-3629

37. Luca L (2015) The Study of Applying a Quality Management Tool for Solving Non-conformities in a Automotive. In: Applied Mechanics and Materials (Vol, 809. Trans Tech Publications Ltd, pp 12571262 In, .

38. Luís Pereira J, Costa M (2019) From NoSQL databases to decision support systems: developing a business intelligence solution. International Journal for Quality Research, 13(4)

39. Lukoševičius $M$, Jaeger $H$ (2009) Reservoir computing approaches to recurrent neural network training. Computer Science Review 3(3):127-149

40. Luo JC, Xing KY, Zhou MC, Li XL, Wang XN (2017) Scheduling of deadlock and failure-prone automated manufacturing systems via hybrid heuristic search. Int J Prod Res 55(11):3283-3293

41. Manyika J, Chui M, Brown B, Bughin J, Dobbs R, Roxburgh C, Byers H, A (2011) Big data: The next frontier for innovation, competition, and productivity. McKinsey Global Institute

42. Maréchaux JL (2006) Combining service-oriented architecture and event-driven architecture using an enterprise service bus. IBM developer works, 1269-1275

43. Marques M, Cunha A, Mohammed WM, Jardim-Gonçalves R, Agostinho C (2019) loT-based Automatic Nonconformity Detection: a Metalworking SME use case. In: Enterprise Interoperability VIII. 
Springer, Cham, pp 155-165

44. Mayer F, Pantförder D (2014) Unterstützung des Menschen in Cyber-Physical-Production-Systems. In: Industrie 4.0 in Produktion, Automatisierung und Logistik. Springer Vieweg, Wiesbaden, pp 481-491

45. Mocnej, J., Pekar, A., Seah, W. K., Papcun, P., Kajati, E., Cupkova, D., ... Zolotova,I. (2021). Qualityenabled decentralised loT architecture with efficient resources utilisation. Robotics and ComputerIntegrated Manufacturing, 67, 102001

46. Moeuf A, Pellerin R, Lamouri S, Tamayo-Giraldo S, Barbaray R (2018) The industrial management of SMEs in the era of Industry 4.0. Int J Prod Res 56(3):1118-1136

47. Mou L, Ghamisi P, Zhu XX (2017) Deep recurrent neural networks for hyperspectral image classification. IEEE Trans Geosci Remote Sens 55(7):3639-3655

48. Müller JM, Buliga O, Voigt KI (2018) Fortune favors the prepared: How SMEs approach business model innovations in Industry 4.0. Technol Forecast Soc Chang 132:2-17

49. Nikolay I (2016) A study on optimisation of nonconformities management cost in the quality management system (QMS) of small-sized enterprise of the construction industry. Procedia Eng 153:228-231

50. Núñez M, Bonhaure D, González M, Cernuzzi L (2020) A model-driven approach for the development of native mobile applications focusing on the data layer. J Syst Softw 161:110489

51. Oakland J (2005) From quality to excellence in the 21st century. Total Quality Management Business Excellence 16(8-9):1053-1060

52. Park J (2015) Evaluating a mobile data-collection system for production information in SMEs. Comput Ind 68:53-64

53. Putnik GD, Varela MLR, Carvalho C, Alves C, Shah V, Castro H, Ávila P (2015) Smart objects embedded production and quality management functions. International Journal for Quality Research 9(1):151-166, ISSN 1800-6450

54. Ramasamy A, Chowdhury S (2020) Big Data Quality Dimensions: A Systematic Literature Review. Journal of Information Systems and Technology Management, 17

55. Ren L, Zhang L, Tao F, Zhao C, Chai X, Zhao X (2015) Cloud manufacturing: from concept to practice. Enterprise Information Systems 9(2):186-209

56. Salkin C, Oner M, Ustundag A, Cevikcan E (2018) A conceptual framework for Industry 4.0. In: Industry 4.0: Managing The Digital Transformation. Springer, Cham, pp 3-23

57. Schmitt J, Bönig J, Borggräfe T, Beitinger G, Deuse J (2020) Predictive model-based quality inspection using Machine Learning and Edge Cloud Computing. Adv Eng Inform 45:101101

58. Schumacher A, Nemeth T, Sihn W (2019) Roadmapping towards industrial digitalisation based on an Industry 4.0 maturity model for manufacturing enterprises. Procedia Cirp 79:409-414

59. Segovia D, Mendoza M, Mendoza E, González E (2015) Augmented reality as a tool for production and quality monitoring. Procedia Computer Science 75:291-300 
60. Singh M, Khan IA, Grover S (2012, October) Tools and techniques for quality management in manufacturing industries. In National Conference on Trends and Advances in Mechanical Engineering (pp. 853-859)

61. Stergiou C, Psannis KE, Kim BG, Gupta B (2018) Secure integration of loT and cloud computing. Future Generation Computer Systems 78:964-975

62. Taiping Mo S Liao \& Wei M (2014) Deadlock-free Production Scheduling with Dynamic Buffers in MES. Smart Science 2:4:196-201. DOI:10.1080/23080477.2014.11665626

63. Tao F, Qi Q, Liu A, Kusiak A (2018) Data-driven smart manufacturing. J Manuf Syst 48:157-169

64. Teixeira, S., Agrizzi, B. A., Pereira Filho, J. G., Rossetto, S., Pereira, I. S. A.,Costa, P. D., ... Martinelli, R. R. (2020). LAURA architecture: Towards a simpler way of building situation-aware and business-aware IoT applications. Journal of Systems and Software, 161, 110494

65. Trappey AJ, Trappey CV, Govindarajan UH, Chuang AC, Sun JJ (2017) A review of essential standards and patent landscapes for the Internet of Things: A key enabler for Industry 4.0. Adv Eng Inform 33:208-229

66. Trentesaux D, Borangiu T, Thomas A (2016) Emerging ICT concepts for smart, safe and sustainable industrial systems

67. Tuertmann R (2016) Challenges and design of a data-oriented complaint and failure management. Total Quality Management Business Excellence 27(8):885 - 896

68. Vernim S, Reinhart G (2016) Usage frequency and user-friendliness of mobile devices in assembly. Procedia CIRP 57:510-515

69. Villalba-Diez J, Schmidt D, Gevers R, Ordieres-Meré J, Buchwitz M, Wellbrock W (2019) Deep learning for industrial computer vision quality control in the printing industry 4.0. Sensors 19(18):3987

70. Wang S, Wan J, Li D, Zhang C (2016) Implementing smart factory of industrie 4.0: an outlook. Int J Distrib Sens Netw 12(1):3159805

71. Wee D, Kelly R, Cattel J, Breunig M (2015) Industry 4.0-how to navigate digitisation of the manufacturing sector. McKinsey \& Company, p 58

72. Xu D, Nageshwaraniyer SS, Son YJ (2016) A service-oriented simulation integration platform for hierarchical manufacturing planning and control. Int J Prod Res 54(23):7212-7230

73. Závadská Z, Závadský J (2018) Quality managers and their future technological expectations related to Industry 4.0. Total Quality Management \& Business Excellence, 1-25

74. Zhang Q, Cheng L, Boutaba R (2010) Cloud computing: state-of-the-art and research challenges. Journal Of Internet Services Applications 1(1):7-18

75. Zhang Y, Ren S, Liu Y, Si S (2017) A big data analytics architecture for cleaner manufacturing and maintenance processes of complex products. J Clean Prod 142:626-641

76. Zhong RY, Xu X, Klotz E, Newman ST (2017) Intelligent manufacturing in the context of industry 4.0: a review. Engineering 3(5):616-630 
77. Zhou K, Liu T, Zhou L (2015, August) Industry 4.0: Towards future industrial opportunities and challenges. In 2015 12th International conference on fuzzy systems and knowledge discovery (FSKD) (pp. 2147-2152). IEEE

\section{Tables}

Table 1. Existing software solutions for NC management

\begin{tabular}{|l|c|c|c|c|c|l|l|l|l|}
\hline Solution & $\begin{array}{l}\text { Suitable for } \\
\text { automotive } \\
\text { industry }\end{array}$ & $\begin{array}{l}\text { Cloud } \\
\text { based }\end{array}$ & $\begin{array}{l}\text { Real- } \\
\text { time }\end{array}$ & $\begin{array}{l}\text { For } \\
\text { mobile } \\
\text { phones }\end{array}$ & $\begin{array}{l}\text { NC } \\
\text {,pattern } \\
\text { catcher” }\end{array}$ & $\begin{array}{l}\text { Statistical } \\
\text { data } \\
\text { modules }\end{array}$ \\
\hline isoTracker & $\mathrm{X}$ & $\mathrm{X}$ & $\mathrm{X}$ & & & & & & \\
rearcode & $\begin{array}{l}\text { Sensors } \\
\text { data } \\
\text { acquisition }\end{array}$ & $\begin{array}{l}\text { Single } \\
\text { programming } \\
\text { language } \\
\text { application }\end{array}$ \\
\hline Intelex & $\mathrm{X}$ & $\mathrm{X}$ & $\mathrm{X}$ & $\mathrm{X}$ & $\mathrm{X}$ & $\mathrm{X}$ & & & \\
\hline Pilgrim & $\mathrm{X}$ & $\mathrm{X}$ & $\mathrm{X}$ & $\mathrm{X}$ & $\mathrm{X}$ & & & & \\
\hline Qualityze & & $\mathrm{X}$ & & $\mathrm{X}$ & & & & & \\
\hline Qualio & $\mathrm{X}$ & $\mathrm{X}$ & $\mathrm{X}$ & & & $\mathrm{X}$ & & & \\
\hline ComplianeQuest & $\mathrm{X}$ & $\mathrm{X}$ & $\mathrm{X}$ & $\mathrm{X}$ & $\mathrm{X}$ & $\mathrm{X}$ & $\mathrm{X}$ & $\mathrm{X}$ & \\
\hline $\begin{array}{l}\text { Solution } \\
\text { presented in } \\
\text { this paper }\end{array}$ & & & & & & & & & $\mathrm{X}$ \\
\hline
\end{tabular}

Table 2 Percentage of intelligent technologies utilisation in NCM (Závadská and Závadský, 2018).

\begin{tabular}{|c|c|c|c|c|c|c|c|c|}
\hline & $\begin{array}{l}\text { Smart } \\
\text { glasses }\end{array}$ & $\begin{array}{l}\text { Smart } \\
\text { gloves }\end{array}$ & $\begin{array}{l}\text { Smart } \\
\text { watches }\end{array}$ & $\begin{array}{l}\text { Smart } \\
\text { phones/ } \\
\text { tablets }\end{array}$ & $\begin{array}{l}\text { RFID } \\
\text { technology }\end{array}$ & Barcode & $\begin{array}{l}\text { QR } \\
\text { code }\end{array}$ & MES \\
\hline $\begin{array}{l}\text { Nonconformity } \\
\text { management }\end{array}$ & 27 & 2 & 50 & 98 & 20 & 75 & 70 & 77 \\
\hline
\end{tabular}

Table 3 Results of software solution utilization in observed organizations 


\begin{tabular}{|c|c|c|c|c|c|c|c|c|c|c|}
\hline & \multicolumn{3}{|l|}{ SME1 } & \multicolumn{3}{|l|}{ SME2 } & \multicolumn{3}{|l|}{ SME3 } \\
\hline & & \multirow{3}{*}{$\frac{\text { before }}{38}$} & \multirow{3}{*}{$\frac{\text { after }}{51}$} & \multirow[t]{2}{*}{$\%$} & \multirow[b]{2}{*}{ before } & \multirow[b]{2}{*}{ after } & \multirow[t]{2}{*}{$\%$} & \multirow[b]{2}{*}{ before } & \multirow[b]{2}{*}{ after } & \multirow[t]{2}{*}{$\%$} \\
\hline & & & & & & & & & & \\
\hline 1 & $\begin{array}{l}\text { Number of } \\
\text { NCs detected }\end{array}$ & & & $34 \%$ é & 41 & 48 & $17 \%$ é & 43 & 52 & $22 \%$ é \\
\hline 2 & $\begin{array}{l}\text { Number of } \\
\text { NCs reported }\end{array}$ & 37 & 45 & $19 \%$ é & 40 & 44 & $8 \%$ é & 43 & 48 & $11 \%$ é \\
\hline 3 & $\begin{array}{l}\text { Number of } \\
\text { preventive } \\
\text { actions taken }\end{array}$ & 12 & 15 & $25 \%$ é & 16 & 19 & $22 \%$ é & 15 & 20 & $29 \%$ é \\
\hline 4 & $\begin{array}{l}\text { Number of } \\
\text { corrective } \\
\text { actions taken }\end{array}$ & 14 & 17 & $17 \%$ é & 14 & 16 & $14 \%$ é & 12 & 14 & $19 \%$ é \\
\hline 5 & $\begin{array}{l}\text { Number of } \\
\text { measures } \\
\text { defined based } \\
\text { on gathered } \\
\text { report }\end{array}$ & 15 & 20 & $31 \%$ é & 17 & 20 & $21 \%$ é & 12 & 18 & $25 \%$ é \\
\hline & $\begin{array}{l}\text { Number of } \\
\text { measures } \\
\text { taken based } \\
\text { on patterns }\end{array}$ & - & 7 & & - & 4 & & - & 3 & \\
\hline 6 & $\begin{array}{ll}\text { Time } & \text { for } \\
\text { reporting } & \text { (in } \\
\text { minutes } & \\
\text { average) } & \end{array}$ & 36 & 18 & $45 \%$ ê & 32 & 19,5 & $39 \%$ ê & 24 & 22,8 & $5 \% 5$ ê \\
\hline 7 & $\begin{array}{l}\text { Time for } \\
\text { definition of } \\
\text { actions taken } \\
\text { (in minutes } \\
\text { average) }\end{array}$ & 48 & 24 & $50 \%$ ê & 38 & 20,9 & $45 \%$ ê & 44 & 22 & $50 \%$ ê \\
\hline 8 & $\begin{array}{l}\text { Number of } \\
\text { employees } \\
\text { who detected } \\
\text { and reported } \\
\text { nonconformity }\end{array}$ & 15 & 36 & $140 \%$ é & 11 & 33 & $200 \%$ é & 20 & 42 & $110 \%$ é \\
\hline
\end{tabular}

Table 4 Number of NCs detected in 6 months before software implementation and six months after software utilization (NCs are related to specific sections of ISO 9001: 2015. 


\begin{tabular}{|c|c|c|c|c|c|c|}
\hline \multirow{2}{*}{$\begin{array}{l}\text { Subsections of the ISO } \\
9001: 2015 \text { standard }\end{array}$} & \multicolumn{3}{|c|}{ Before software utilization } & \multicolumn{3}{|c|}{ After software utilization } \\
\hline & SME 1 & SME 2 & SME 3 & SME 1 & SME 2 & SME 3 \\
\hline 4.1 & 0 & 0 & 0 & 0 & 0 & 0 \\
\hline 4.2 & 0 & 0 & 1 & 2 & 1 & 3 \\
\hline 4.3 & 2 & 2 & 2 & 3 & 3 & 3 \\
\hline 4.4 & 2 & 1 & 2 & 1 & 2 & 3 \\
\hline 5.1 & 0 & 0 & 0 & 0 & 0 & 0 \\
\hline 5.2 & 0 & 0 & 0 & 0 & 0 & 0 \\
\hline 5.3 & 2 & 2 & 3 & 3 & 3 & 4 \\
\hline 6.1 & 2 & 2 & 3 & 3 & 3 & 4 \\
\hline 6.2 & 3 & 3 & 2 & 4 & 4 & 3 \\
\hline 6.3 & 0 & 0 & 0 & 0 & 0 & 0 \\
\hline 7.1 & 0 & 0 & 0 & 0 & 0 & 0 \\
\hline 7.1 .1 & 0 & 0 & 0 & 0 & 0 & 0 \\
\hline 7.1 .2 & 0 & 0 & 0 & 0 & 0 & 0 \\
\hline 7.1 .3 & 2 & 3 & 3 & 4 & 3 & 4 \\
\hline 7.1 .4 & 0 & 0 & 0 & 0 & 0 & 0 \\
\hline 7.1 .5 & 1 & 1 & 2 & 3 & 2 & 3 \\
\hline 7.1 .6 & 0 & 0 & 0 & 0 & 0 & 0 \\
\hline 7.2 & 1 & 1 & 3 & 2 & 2 & 2 \\
\hline 7.3 & 0 & 0 & 0 & 0 & 0 & 0 \\
\hline 7.4 & 0 & 0 & 1 & 0 & 0 & 0 \\
\hline 7.5 & 2 & 2 & 1 & 0 & 0 & 0 \\
\hline 8.1 & 0 & 0 & 0 & 0 & 0 & 0 \\
\hline 8.2 & 2 & 2 & 0 & 0 & 0 & 0 \\
\hline 8.3 & 0 & 0 & 2 & 0 & 0 & 0 \\
\hline 8.4 & 2 & 3 & 1 & 4 & 3 & 6 \\
\hline 8.5 & 4 & 7 & 4 & 8 & 8 & 7 \\
\hline 8.6 & 2 & 1 & 1 & 2 & 1 & 0 \\
\hline 8.7 & 4 & 4 & 3 & 7 & 6 & 5 \\
\hline 9.1 & 2 & 2 & 2 & 1 & 2 & 2 \\
\hline 9.2 & 1 & 2 & 2 & 2 & 2 & 1 \\
\hline 9.3 & 2 & 2 & 3 & 2 & 2 & 2 \\
\hline 10.1 & 0 & 0 & 0 & 0 & 1 & 0 \\
\hline 10.2 & 2 & 1 & 2 & 0 & 0 & 0 \\
\hline 10.3 & 0 & 0 & 0 & 0 & 0 & 0 \\
\hline TOTAL & 38 & 41 & 43 & 51 & 48 & 52 \\
\hline
\end{tabular}

Table 5. Insert, Update and Select queries per second for each considered technology 


\begin{tabular}{|c|c|}
\hline MongoDB Insert syntax sample & MariaDB/MySQL Insert syntax sample \\
\hline 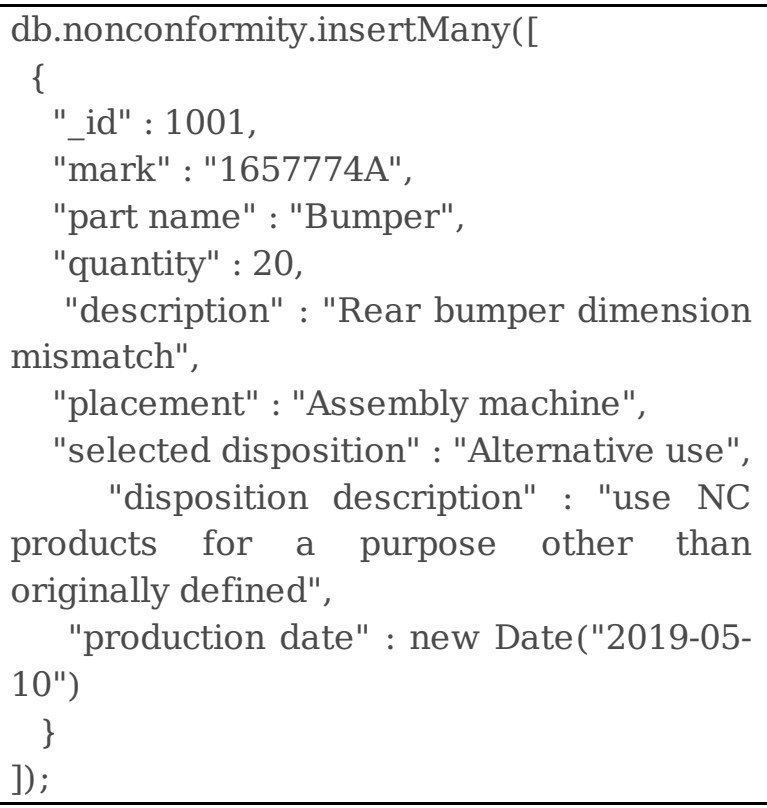 & $\begin{array}{l}\text { INSERT INTO nonconformity VALUES } \\
\text { (1001, '1657774A', 'Bumper', 20, } \\
\text { 'Rear bumper dimension mismatch', 'Assembly machine', } \\
\text { 'Alternative use', 'use NC products for a purpose other than } \\
\text { originally defined', '2019-05-10') }\end{array}$ \\
\hline MongoDB Update syntax sample & MariaDB/MySQL Update syntax sample \\
\hline $\begin{array}{l}\text { db.nonconformity.update( } \\
\text { \{_id: } 1001\}, / / \text { specifies which document } \\
\text { to update } \\
\text { \{ } \\
\quad \text { \$inc: }\{\text { quantity: } 25\}, / / \text { increments the } \\
\text { field value } \\
\quad \text { \$set: }\{/ / \text { replaces the field value } \\
\quad \text { "selected disposition" : "Return to the } \\
\text { supplier", } \\
\quad \text { "disposition description" : "Products } \\
\text { have to be returned to the supplier since } \\
\text { they are damaged." } \\
\} \\
\}\end{array}$ & $\begin{array}{l}\text { UPDATE nonconformity } \\
\text { SET quantity = quantity + } 5 \\
\quad \text { selected disposition = "Return to the supplier", } \\
\quad \text { disposition description = "Products have to be returned to } \\
\text { the supplier since they are damaged" } \\
\text { WHERE_id = } 1001\end{array}$ \\
\hline MongoDB select syntax sample & MariaDB/MySQL select syntax sample \\
\hline db. nonconformity.find( \{\} ) & SELECT * FROM nonconformity \\
\hline
\end{tabular}

Table 6. Insert queries per second for each considered technology

\begin{tabular}{|l|l|l|l|l|l|l|}
\hline Previous technologies & 500 & $\mathbf{1 0 0 0} \mathbf{q}$ & $\mathbf{2 5 0 0} \mathrm{q}$ & $\mathbf{5 0 0 0}$ & $\mathbf{7 5 0 0}$ & $\mathbf{1 0 0 0 0}$ \\
\hline MySQL/Apache/PHP & 0.0800 & 0.1638 & 0.4019 & 0.8014 & 1.2037 & 1.6027 \\
\hline MariaDB/Apache/PHP & 0.0692 & 0.1439 & 0.3479 & 0.6931 & 1.0385 & 1.3846 \\
\hline MEAN & 0.0224 & 0.0660 & 0.2323 & 0.3671 & 0.6284 & 0.9271 \\
\hline
\end{tabular}

Table 7. Update queries per second for each considered technology

\begin{tabular}{|l|l|l|l|l|l|l|}
\hline Previous technologies & $\mathbf{5 0 0}$ & $\mathbf{1 0 0 0}$ & $\mathbf{2 5 0 0}$ & $\mathbf{5 0 0 0}$ & $\mathbf{7 5 0 0}$ & $\mathbf{1 0 0 0 0}$ \\
\hline MySQL/Apache/PHP & 0.0697 & 0.1552 & 0.3873 & 0.8789 & 1.4402 & 2.0828 \\
\hline MariaDB/Apache/PHP & 0.0783 & 0.1445 & 0.2722 & 0.3427 & 0.5177 & 0.6900 \\
\hline MEAN & 0.0106 & 0.0525 & 0.2023 & 0.3254 & 0.3677 & 0.4368 \\
\hline
\end{tabular}


Table 8. Select queries per second for each considered technology

\begin{tabular}{|l|l|l|l|l|l|l|}
\hline Previous technologies & $\mathbf{5 0 0}$ & $\mathbf{1 0 0 0}$ & $\mathbf{2 5 0 0}$ & $\mathbf{5 0 0 0}$ & $\mathbf{7 5 0 0}$ & $\mathbf{1 0 0 0 0}$ \\
\hline MySQL/Apache/PHP & 0.0394 & 0.0732 & 0.1965 & 0.3943 & 0.6011 & 0.7999 \\
\hline MariaDB/Apache/PHP & 0.0306 & 0.0655 & 0.1696 & 0.3385 & 0.5156 & 0.6826 \\
\hline MEAN & 0.0037 & 0.0328 & 0.1116 & 0.1832 & 0.3110 & 0.4597 \\
\hline
\end{tabular}

\section{Footnotes}

[1]TypeScript presents a type-checking superset of JavaScript that compiles to JavaScript.

[2]Web Bluetooth is an API used to discover and communicate with devices over the Bluetooth 4.

[3]V8 is the runtime environment for JavaScript created for Google Chrome. It is free software since 2008, written in $\mathrm{C}++$ with the purpose to compile the JavaScript source code into machine code instead of interpreting it in real-time.

[4]Libuv is a library that supports asynchronous $1 / 0$ originally written for Node.js, know also used by other platforms.

[5] before Node.js establishment, it was necessary to be familiar with, at least, two programming languages, one used for the presentation, and the other for the business tier development.

\section{Figures}




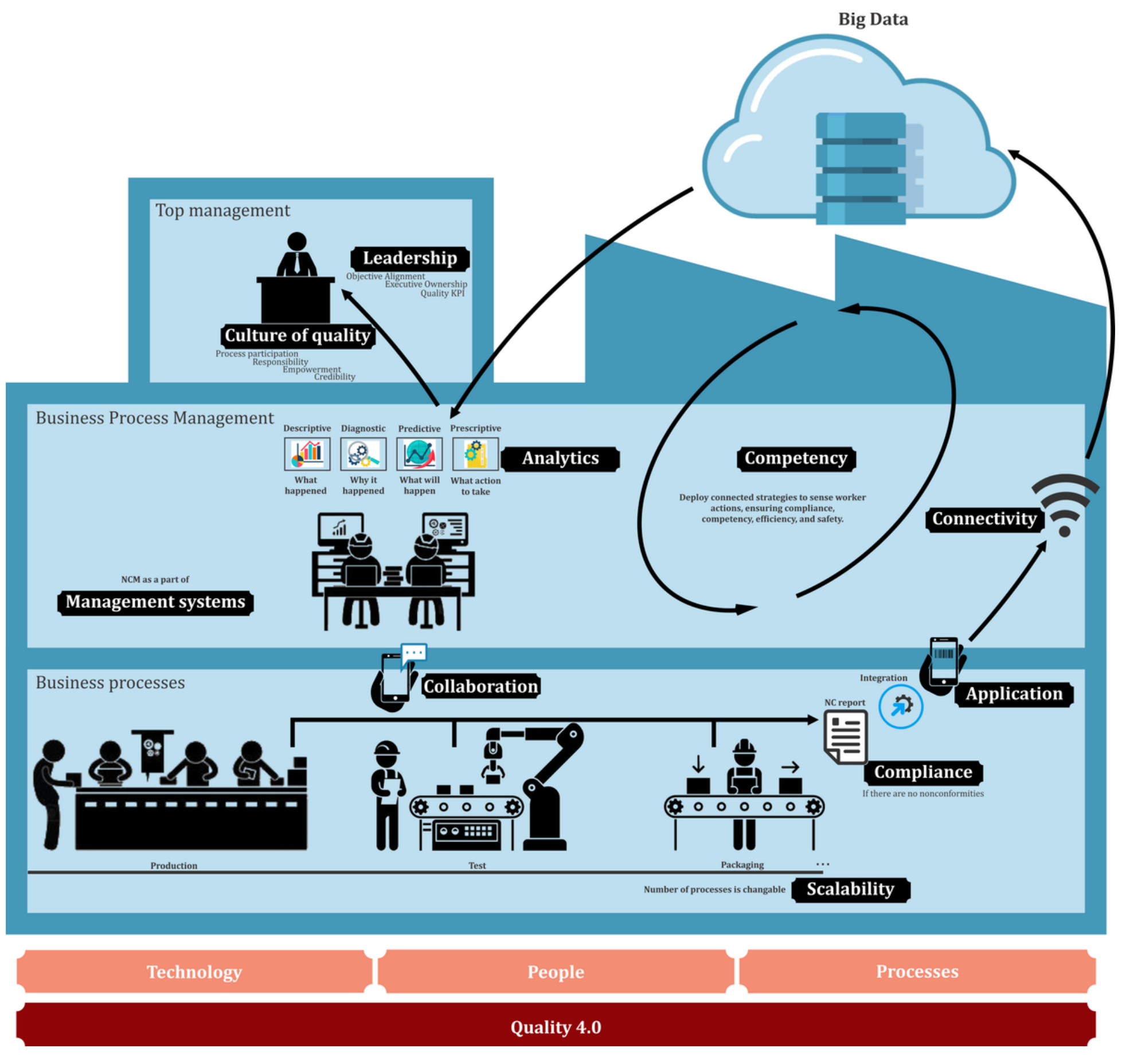

Figure 1

Quality 4.0 pillars adaptation 
Presentation tier

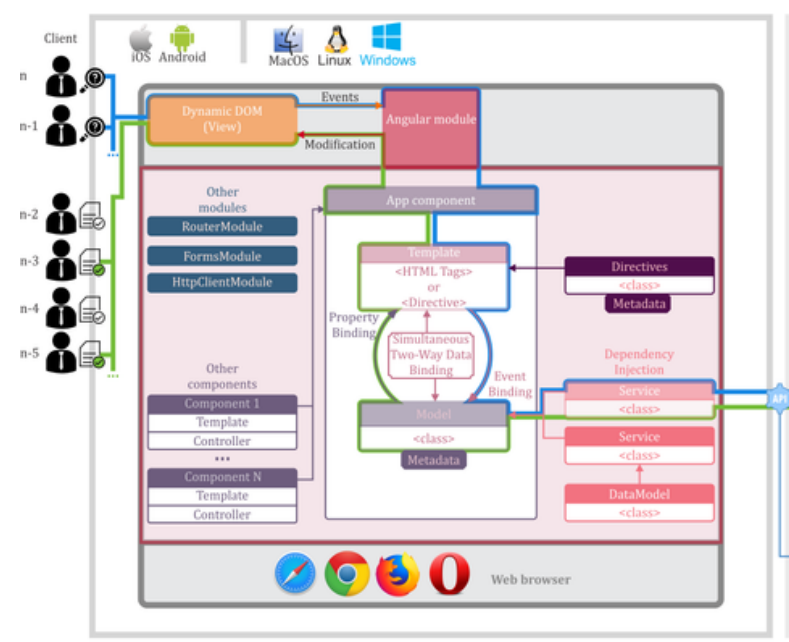

Angular 8.0

\section{Business tier}

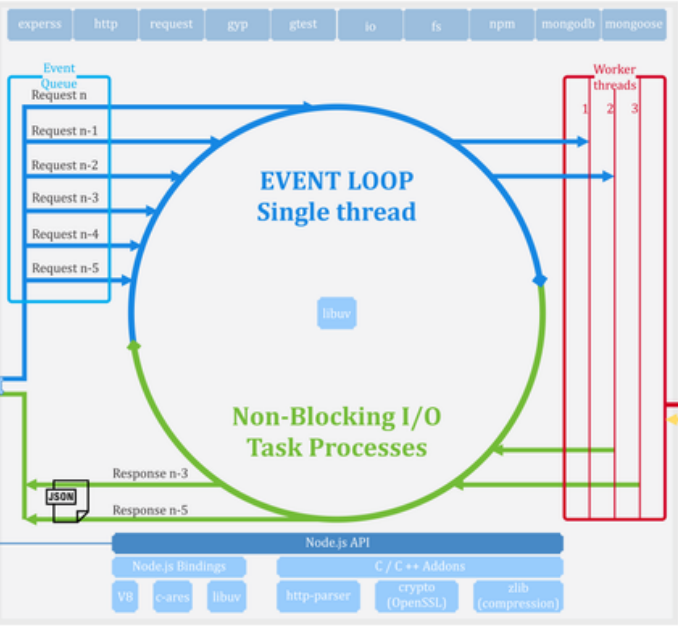

node (s)
Database tier

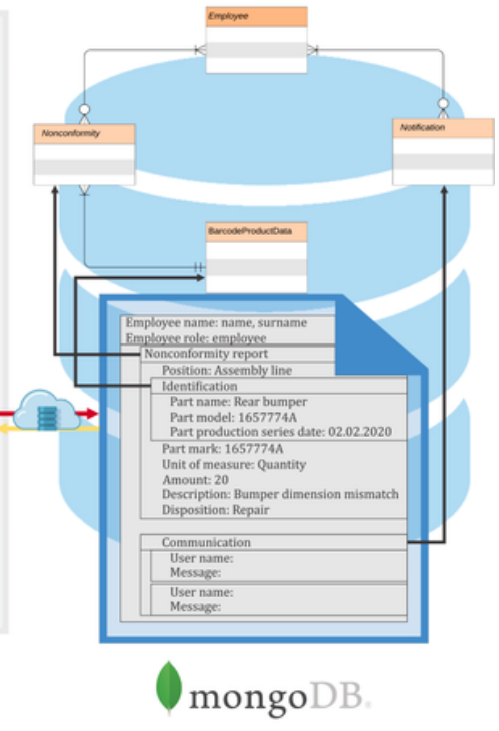

Figure 2

Data flow through adopted technologies

Cloud server

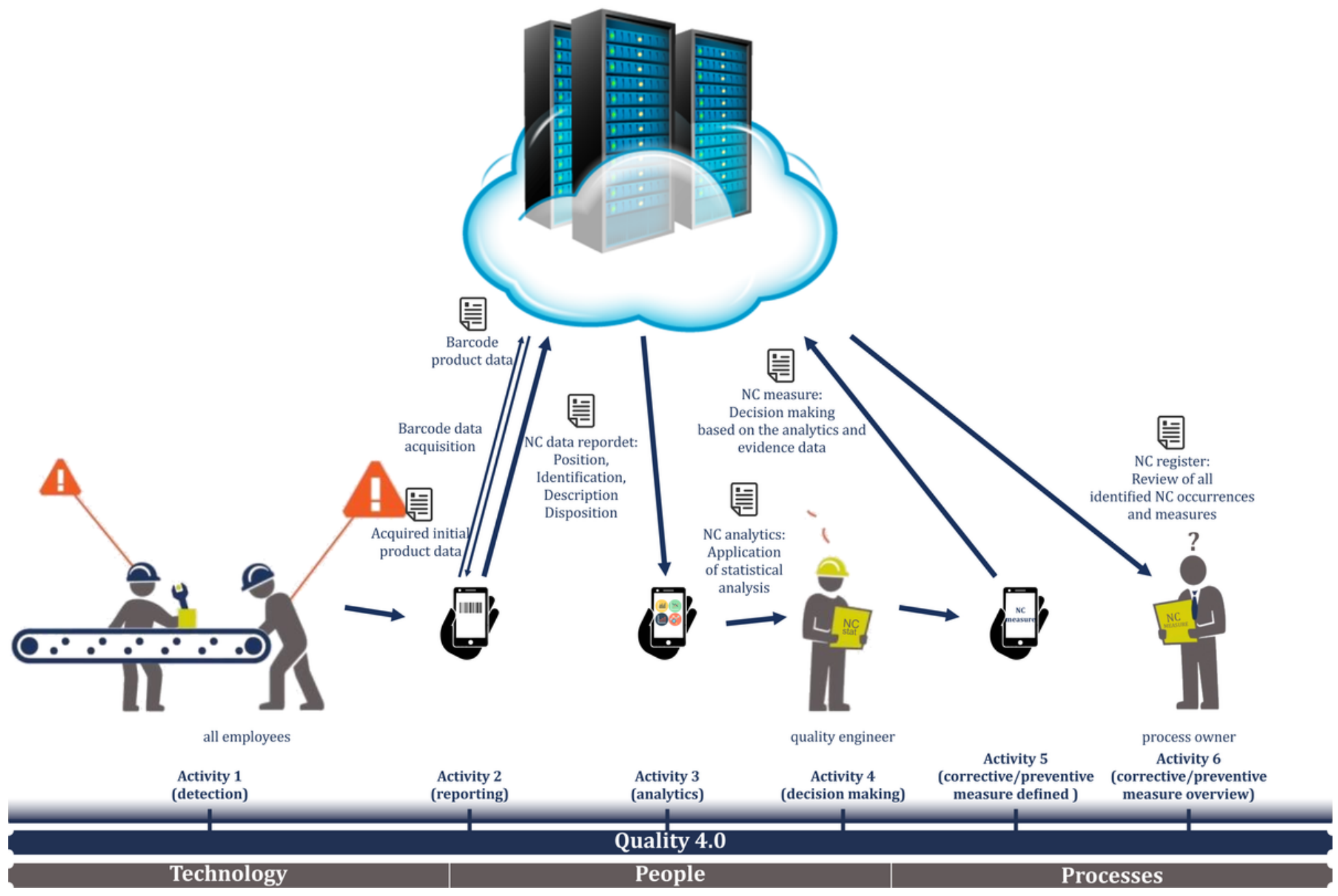


Figure 3

Illustration of activities within NCM workflow

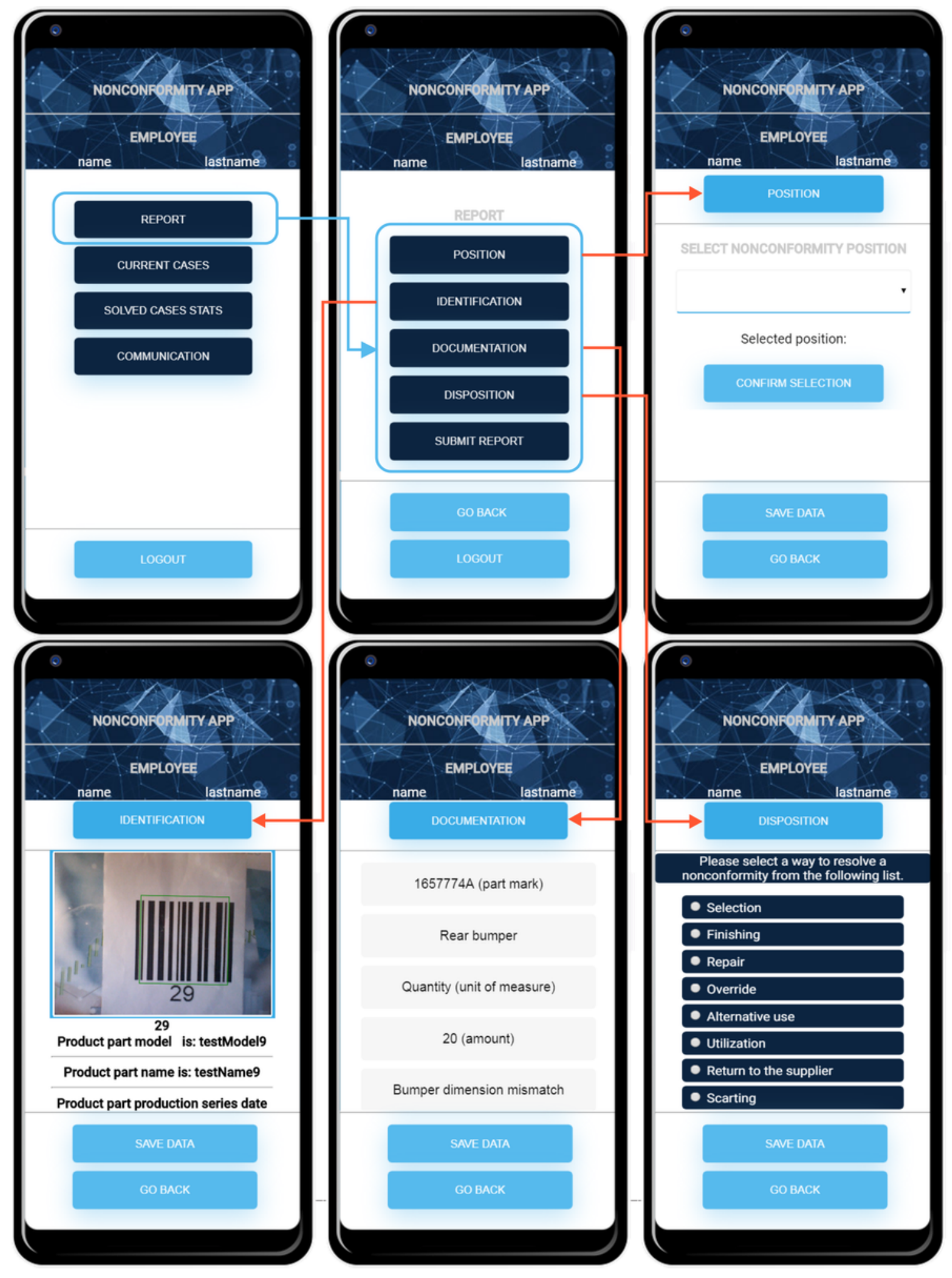

Figure 4

Employee user interface with defined functionalities 

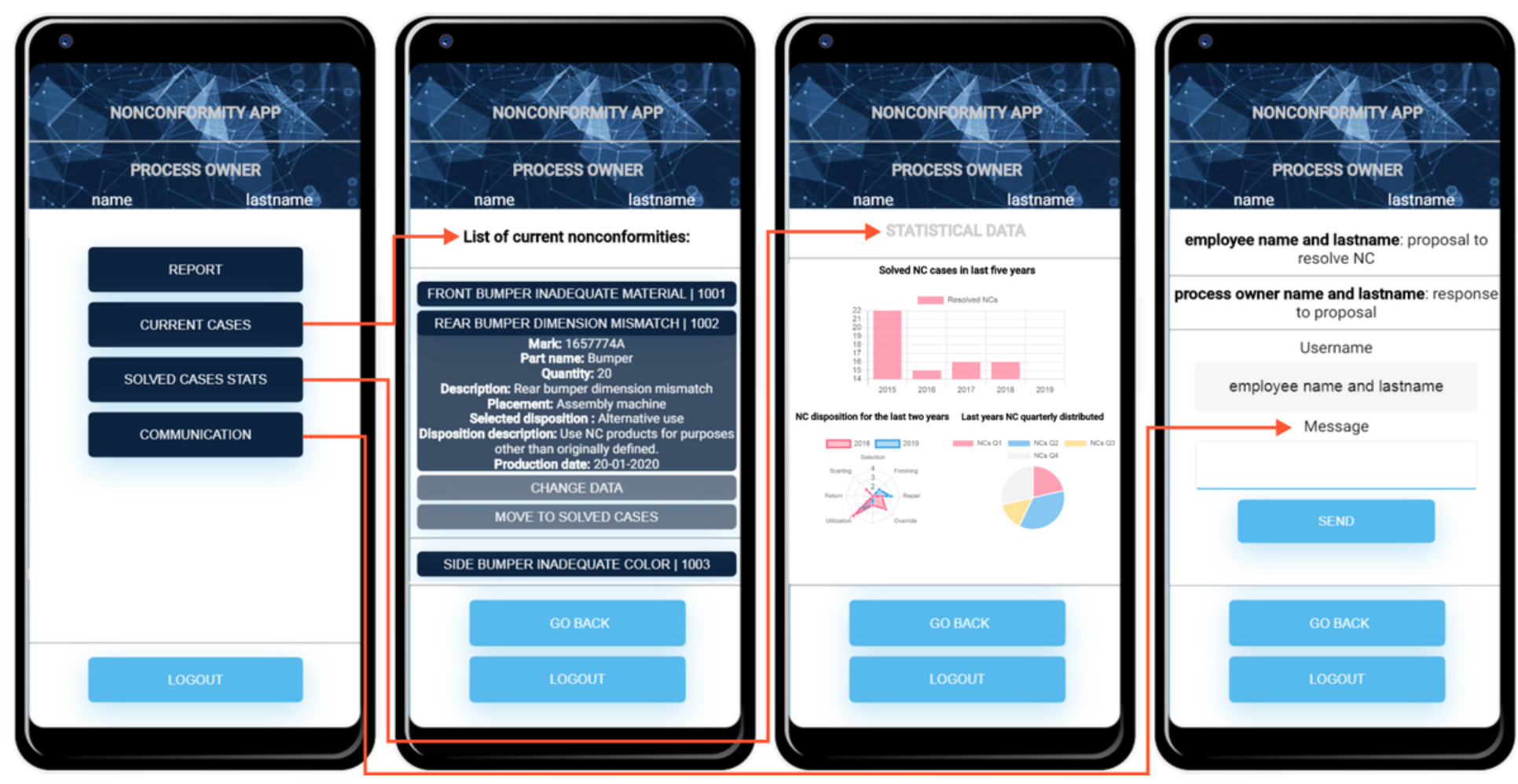

Figure 5

Process owner user interface with defined functionalities 


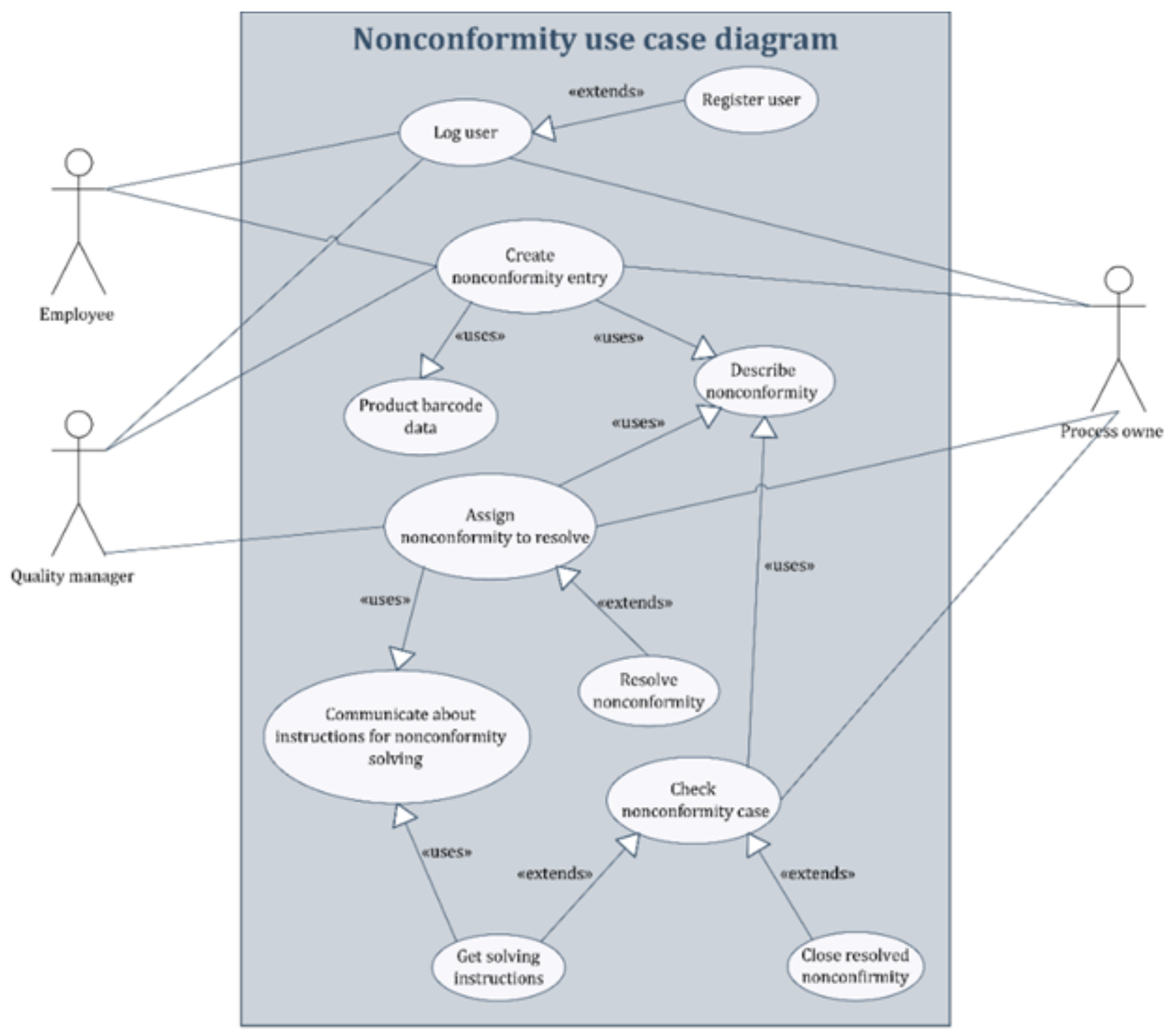

Figure 6

A use case diagram for $\mathrm{NC}$ reporting software solution applied in automotive industry SMEs 


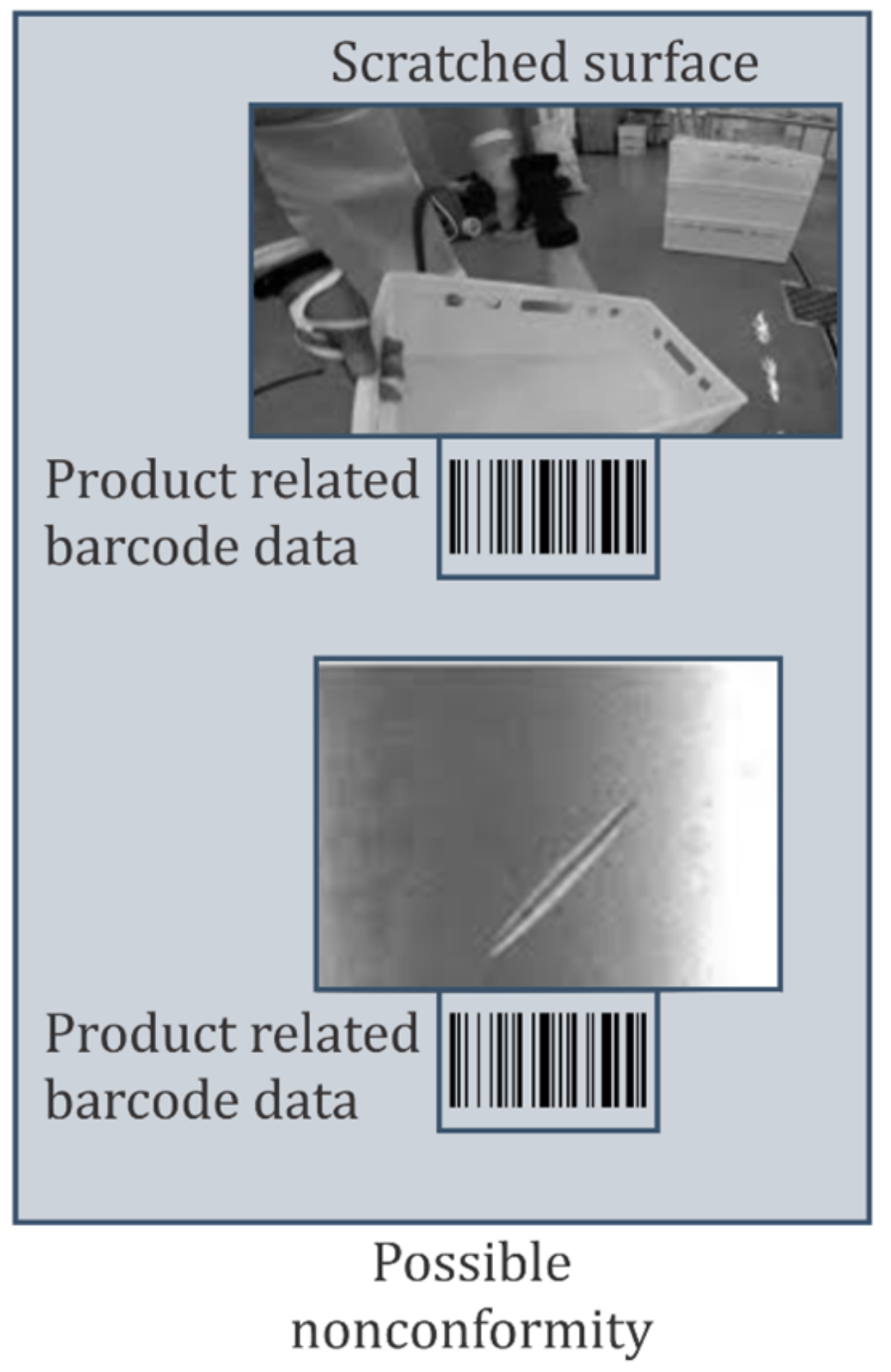

Figure 7

Possible nonconformities 